\title{
Structural and magnetic properties of amorphous Co-W alloyed nanoparticles
}

\author{
A. I. Figueroa, ${ }^{1,2, *}$ J. Bartolomé, ${ }^{1,2}$ L. M. García, ${ }^{1,2}$ F. Bartolomé, ${ }^{1,2}$ C. Magén,,${ }^{2,3,4}$ A. Ibarra, ${ }^{3}$ L. Ruiz, ${ }^{5}$ J. M. González-Calbet, ${ }^{5}$ \\ F. Petroff, ${ }^{6}$ C. Deranlot, ${ }^{6}$ S. Pascarelli, ${ }^{7}$ P. Bencok, ${ }^{7}$ N. B. Brookes, ${ }^{7}$ F. Wilhelm, ${ }^{7}$ and A. Rogalev ${ }^{7}$ \\ ${ }^{1}$ Instituto de Ciencia de Materiales de Aragón (ICMA), CSIC-Universidad de Zaragoza, E-50009 Zaragoza, Spain \\ ${ }^{2}$ Departamento de Física de la Materia Condensada, Universidad de Zaragoza, E-50009 Zaragoza, Spain \\ ${ }^{3}$ Instituto de Nanociencia de Aragón (INA), Universidad de Zaragoza, E-50018 Zaragoza, Spain \\ ${ }^{4}$ Fundación ARAID, E-50004 Zaragoza, Spain \\ ${ }^{5}$ Departamento de Química Inorgánica, Universidad Complutense de Madrid, E-28040 Madrid, Spain \\ ${ }^{6}$ Unité Mixte de Physique CNRS/Thales, F-91767 Palaiseau Cedex, France and Université Paris-Sud, F-91405 Orsay Cedex, France \\ ${ }^{7}$ European Synchrotron Radiation Facility (ESRF), F-38043 Grenoble, France
}

(Received 15 April 2011; revised manuscript received 3 October 2011; published 16 November 2011)

\begin{abstract}
W-capped Co nanoparticles dispersed in an alumina matrix are studied by means of high-resolution transmission electron microscopy, extended x-ray absorption fine structure, SQUID-based magnetic measurements, dc magnetization, ac magnetic susceptibility, and x-ray magnetic circular dichroism. Results show the formation of amorphous $\mathrm{Co}-\mathrm{W}$ alloy nanoparticles, the magnetic properties of which are modified by the amount of $\mathrm{W}$ or $\mathrm{Co}$ present in the samples. The average Co magnetic moment depends on the number of $\mathrm{W}$ atoms surrounding it. Co-W particles show superparamagnetic behavior and are described as an array of noninteracting particles with random anisotropy axes and an average moment per particle proportional to the particle volume and to the average Co moment for each alloy composition. Values of the magnetic anisotropy constant of the particles are on the order of $10^{6} \mathrm{erg} / \mathrm{cm}^{3}$, higher than that of bulk Co. Evidence of short-range ordering within each amorphous particle is found that provides insight of the origin of their magnetic anisotropy.
\end{abstract}

DOI: 10.1103/PhysRevB.84.184423

PACS number(s): 75.75.Fk, 75.50.Kj, 75.30.Gw, 61.46.Df

\section{INTRODUCTION}

Advances in the synthesis and processing of materials at the nanoscale during the last two decades have made it possible to fabricate high-quality samples of magnetic nanoparticles (NPs) with uniform material properties ${ }^{1,2}$ and evenly self-organized lattices of NPs. ${ }^{2,3}$ These systems have received attention for fundamental research because they are ideal systems to study the properties of single particles as well as their mutual interactions. ${ }^{4-8}$ For applications, the understanding and tailoring of the magnetic properties such as anisotropy or dipolar interactions between particles is also crucial (for instance, in fields such as high-density recording media).$^{9}$ In this context, we have performed over the last years an extensive work on self-organized lattices of nanometersized Co magnetic clusters $(1-4 \mathrm{~nm})$ prepared by sequential deposition of $\mathrm{Al}_{2} \mathrm{O}_{3}$ and Co layers on a Si substrate. In these granular systems, we have been able to control the cluster size ${ }^{10}$ the average number of neighbors to a given cluster, ${ }^{8}$ and even the surface anisotropy by capping the cobalt nanoparticles with noble metals such as $\mathrm{Cu}, \mathrm{Ag}$, and $\mathrm{Au} .{ }^{11}$ These studies have enabled us to perform quantitative determination of parameters such as magnetic anisotropy ${ }^{12,13}$ or dipolar interactions. ${ }^{8}$ The capping metal does not diffuse on $\mathrm{Co}$, so the Co particle retains its integrity as a nearly spherical crystalline particle. We have demonstrated that there is an enhancement of surface anisotropy with respect to the bare Co NPs, directly correlated with the changes in the orbital moment for the Co surface atoms induced by the surrounding. ${ }^{14}$

To prove this point, magnetization and ac susceptibility measurements, which give information on the magnetic anisotropy, have been complemented with x-ray magnetic circular dichroism (XMCD) spectroscopy, which supplies the average orbital moment of the Co NPs. Our previous work and others have related the increase of the NP magnetocrystalline anisotropy energy with orbital moment anisotropy at the NPs surface, as was proposed by Bruno. ${ }^{15}$

Aside from those surface effects described above for the metal-capped Co NPs, the magnetic anisotropy of NP systems may be intrinsic, such as that identified in chemically ordered CoPt NPs. ${ }^{16}$ The anisotropy of these particles is correlated with the Co orbital anisotropy, which by hybridization with $\mathrm{Pt}$, and due to the high spin-orbit coupling of the latter, gives rise to an enhanced anisotropy, albeit much smaller than in the bulk CoPt $L 1_{0}$ phase. Therefore, alloying Co with other metals may be a method of controlling the intrinsic anisotropy, and XMCD the right technique to probe the modification of the average orbital moment. Tungsten (electron configuration $\left.[\mathrm{Xe}] 4 f^{14} 5 d^{4} 6 s^{2}\right)$ is a good candidate as it may hybridize with Co (electron configuration $[\mathrm{Ar}] 3 d^{7} 4 s^{2}$ ) since it is known to alloy with $\mathrm{Co},{ }^{17}$ and has a less than half-filled $5 d$ band.

Amorphous NPs constitute a different type with variable anisotropy. Few works in this field have been previously performed. Most of them are focused on Fe-based NPs (Refs. 4,18-27) and some on Ni-based NPs, ${ }^{28-30}$ where surface effects are substantial for the blocking process and magnetic anisotropy, while, to our knowledge, no study on the mechanism of intrinsic anisotropy has been so far performed.

This paper is devoted to investigate the open question as to whether amorphous alloys may support intrinsic anisotropy, induced by alloying, irrespective of surface effects. We present a systematic study of structural and magnetic properties of selforganized lattices of Co NPs, this time capped with tungsten. The election of $\mathrm{W}$ is based on the known affinity to produce $\mathrm{Co}-\mathrm{W}$ alloys by sputtering, at room temperature, ${ }^{17,31-33}$ on one hand, and the tendency of these alloys to become amorphous. ${ }^{31,34-36}$ Besides, short-range structural ordering, 
observed in films and bulk amorphous alloys, ${ }^{37,38}$ has been proposed as the mechanism inducing anisotropy in those systems. So, there was a good chance that Co-W alloying would allow varying the NPs anisotropy as a function of the tungsten concentration, as we prove below.

Moreover, since the sequential alumina-Co-W deposition technique employed allows us to obtain self-organized arrays of particles with controlled geometries, i.e., particle sizes and interparticle distances, we have pursued the objective of expanding this potential to study the magnetic properties of amorphous Co-W NPs.

The paper is organized as follows. We first describe the samples studied in Sec. II and the characterization techniques in Sec. III. Then, Sec. IV describes the morphological properties of the Co-W NPs studied by high-resolution transmission electron microscopy (HRTEM) and high-angle annular dark-field scanning transmission electron microscopy (HAADF STEM). The structural properties of the nanoparticles studied via X-ray absorption (XAS) and extended x-ray absorption fine structure (EXAFS) are presented in Sec. V. We discuss the magnetic properties of the system studied by SQUID magnetometry and x-ray magnetic circular dichroism (XMCD) in Secs. VI and VII, respectively. Finally, we summarize our results in Sec. VIII.

\section{SAMPLES STUDIED}

The samples studied were prepared at the Unité Mixte de Physique CNRS/Thales by sequential sputtering deposition of $\mathrm{Al}_{2} \mathrm{O}_{3} \mathrm{Co}$, and $\mathrm{W}$ on a $\mathrm{Si}$ substrate. The alumina, cobalt, and tungsten are deposited using Ar plasma; the metals in dc mode, and the insulator at a rf power of $2.2 \mathrm{~W} / \mathrm{cm}^{2}$. The substrate temperature is kept constant at $293 \mathrm{~K}$ and the Ar pressure is $2 \times$ $10^{-3}$ Torr. The formation of Co aggregates on the amorphous alumina is the result of three-dimensional growth because of the different surface energies between alumina and Co. ${ }^{39}$ Aggregation occurs below a certain threshold of the nominal thickness of $\mathrm{Co}\left(t_{\mathrm{Co}}\right)$ that the layer would have if it were continuous. Previous studies show that not all the Co deposited on the alumina layer aggregates as forming the particles, but there exists a fraction of Co atoms or small clusters dispersed on the matrix, defined as $x_{\text {para }}{ }^{10}$ The values of $x_{\text {para }}$ depend on $t_{\mathrm{Co}}$, varying between 0.5 and 0.13 for $t_{\mathrm{Co}}=0.4$ and $1.0 \mathrm{~nm}$, respectively. ${ }^{10}$ The Co clusters are subsequently capped with a W layer, the nominal thickness of which is denoted by $t_{\mathrm{W}}$. A new alumina layer of about $3 \mathrm{~nm}$ is deposited on top of this $\mathrm{Co}-\mathrm{W}$ system. This sequential deposition process is repeated $N$ times in order to get a multilayer system, following the formula $\mathrm{Al}_{2} \mathrm{O}_{3} /\left(\mathrm{Al}_{2} \mathrm{O}_{3} / \mathrm{Co} / \mathrm{W}\right)_{N} / \mathrm{Al}_{2} \mathrm{O}_{3}$.

The samples measured may be grouped into two series: $a$, those with $t_{\mathrm{Co}}$ fixed at $0.7 \mathrm{~nm}$ and varying $\mathrm{W}$ capping layer, $t_{\mathrm{W}}=0.6,1.5$, and $4.5 \mathrm{~nm}$; and $b$, with constant capping layer $t_{\mathrm{W}}=1.5 \mathrm{~nm}$ and varying $t_{\mathrm{Co}}=0.4,0.7$, and $1.0 \mathrm{~nm}$. Most of the samples have $N=25$, except that with $t_{\mathrm{Co}}=1.0 \mathrm{~nm}$ and $t_{\mathrm{W}}=1.5 \mathrm{~nm}$, for which $N=20$.

\section{EXPERIMENTAL DETAILS}

HRTEM measurements were performed on both $a$ and $b$ samples series in cross-section configuration in a JEOL JEM
3000 F field emission microscope fitted with an Oxford LINK electron dispersive spectrometry (EDS) analyzer at the Universidad Complutense de Madrid. Cross-section specimens were prepared by conventional mechanical thinning and ion milling techniques.

HRTEM and HAADF STEM measurements were performed on two samples in plan-view configuration in a FEI Tecnai ${ }^{\mathrm{TM}}$ G2 F30 STWIN field emission microscope at the Instituto de Nanociencia de Aragón. Samples for plan-view observation were prepared by deposition of a $\mathrm{Al}_{2} \mathrm{O}_{3} / \mathrm{Co} / \mathrm{W} / \mathrm{Al}_{2} \mathrm{O}_{3}$ sandwich on TEM carbon grids, with $t_{\mathrm{Co}}=0.7 \mathrm{~nm}$ and $t_{\mathrm{W}}=0.6$ and $1.5 \mathrm{~nm}$.

XAS and EXAFS spectra for both $a$ and $b$ samples series were measured at the Co $K(7709 \mathrm{eV})$ and $\mathrm{W} L_{3}(10207 \mathrm{eV})$ edges using a double-crystal $\mathrm{Si}(111)$ monocromator in the BM29 beamline at the European Synchrotron Radiation Facility (ESRF). Measurements were performed at $300 \mathrm{~K}$ in fluorescence detection mode with a 13-element Ge solid-state detector with digital signal processing for fluorescence XAS, high-energy resolution, and high count rate. Reference samples were also measured at different edges: Co particles embedded in alumina matrix (referred in the following as "bare Co NPs") $\left(\mathrm{Al}_{2} \mathrm{O}_{3} / \mathrm{Co}\right)_{20}$ with $t_{\mathrm{Co}}=0.7 \mathrm{~nm}$ measured at the Co $K$ edge, $\mathrm{Co}_{3} \mathrm{~W}$ alloy measured at the $\mathrm{Co} K$ and $\mathrm{W} L_{3}$ edges, Au capped Co particles with formula $\left(\mathrm{Al}_{2} \mathrm{O}_{3} / \mathrm{Co} / \mathrm{Au}\right)_{25}, t_{\mathrm{Au}}=1.5$ and $4.5 \mathrm{~nm}$, measured at the $\mathrm{Co} K$ and $\mathrm{Au} L_{3}$ edges. The reference foils are as follows: Co metallic foil measured at the Co $K$ edge, $\mathrm{W}$ metallic foil measured at the $\mathrm{W} L_{3}$ edge, and $\mathrm{Au}$ foil measured at the $\mathrm{Au} L_{3}$ edge. For each edge measured on each sample, four spectra were taken in order to have enough statistics in data treatment, following a $4 \mathrm{~s}$ /point count.

Some of the x-ray absorption near edge structure (XANES) spectra at the $\mathrm{W} L_{3}$ edge were recorded at the ESRF ID12 beamline by a fluorescence detector in backscattering geometry. The APPLE-II undulator and a double-Si-(111)crystal monocromator were used to collect the spectra.

The magnetic study of both $a$ and $b$ samples series was performed by $\chi_{\mathrm{dc}}(T), \chi_{\mathrm{ac}}(T)$, and $M(H)$ measurements in a commercial SQUID magnetometer at the Servicio de Instrumentación Científica of the Universidad de Zaragoza. $\chi_{\mathrm{dc}}(T)$ was measured after zero-field cooling (ZFC) and field cooling (FC) the sample. Temperature was varied between 5 and $150 \mathrm{~K}$, and a 200 Oe field was applied. $M(H)$ measurements were performed at $T \approx 3 T_{B}\left(T_{B}\right.$, blocking temperature of the superparamagnetic particles) under applied fields up to $50 \mathrm{kOe}$. $\chi_{\mathrm{ac}}(T)$ was measured by applying an ac field of 4 Oe in amplitude to the sample with frequency $\omega / 2 \pi$ in between 1 and $480 \mathrm{~Hz}$.

The XAS and XMCD experiments at the Co $L_{2,3}$ edges were performed at the ID08 beamline of the ESRF. An APPLE-II undulator and a spherical grating monochromator were used. The degree of polarization was $\sim 100 \%$. The total electron yield (TEY) detection method was employed. The beam direction and parallel magnetic field applied had an incident angle of $10^{\circ}-15^{\circ}$ with respect to the normal to the plate sample. The XMCD signal was obtained by a direct difference of the XAS spectra recorded with opposite helicities at each magnetic field value and for both orientations of the field. The applied field was $20 \mathrm{kOe}$ and the temperature was $T=7 \mathrm{~K}$ in all cases. 


\section{MORPHOLOGICAL STUDY: MICROSCOPY MEASUREMENTS}

Low-magnification studies in cross-section configuration for all five samples confirmed the presence of 25 layers (20 layers in the case of $t_{\mathrm{Co}}=1.0 \mathrm{~nm}$ and $t_{\mathrm{W}}=1.5 \mathrm{~nm}$ ) in agreement to the nominal layer preparation. HRTEM images and EDS analysis for both samples series (Figs. 1 and 2) indicate that light gray layers correspond to alumina and darker layers to metal (Co and $\mathrm{W}$ ). These layers exhibit a washboard cross-sectional shape. In stark contrast to Co-nanoparticles systems covered with a noble metal layer, where the crystal structure of the Co particle is not affected by the capping, ${ }^{14}$ W alloys with Co in those places where the Co NPs were originally formed. By inspection of the particles in Figs. 1(a) and $1(b)$, there seems to be very-short-range order within each layer due to the higher $\mathrm{Co} / \mathrm{W}$ ratio in these two samples, but this is neither detected by selected area electron diffraction (SAED) nor by Fourier transform. In fact, the SAED images show the typical halo diffraction pattern corresponding to an amorphous structure [see Fig. 2(d)]. Regarding this feature, some authors suggest that amorphous Co-W films consist of tetrahedral units composed of one $\mathrm{W}$ and three Co atoms, which arrange in a structure that does not exhibit macroscopic crystal symmetry. ${ }^{40}$ Others ascribe this short-range ordering (SRO) in amorphous alloys to the existence of icosahedral and octahedral clusters. ${ }^{41}$ Some of these structures are probably

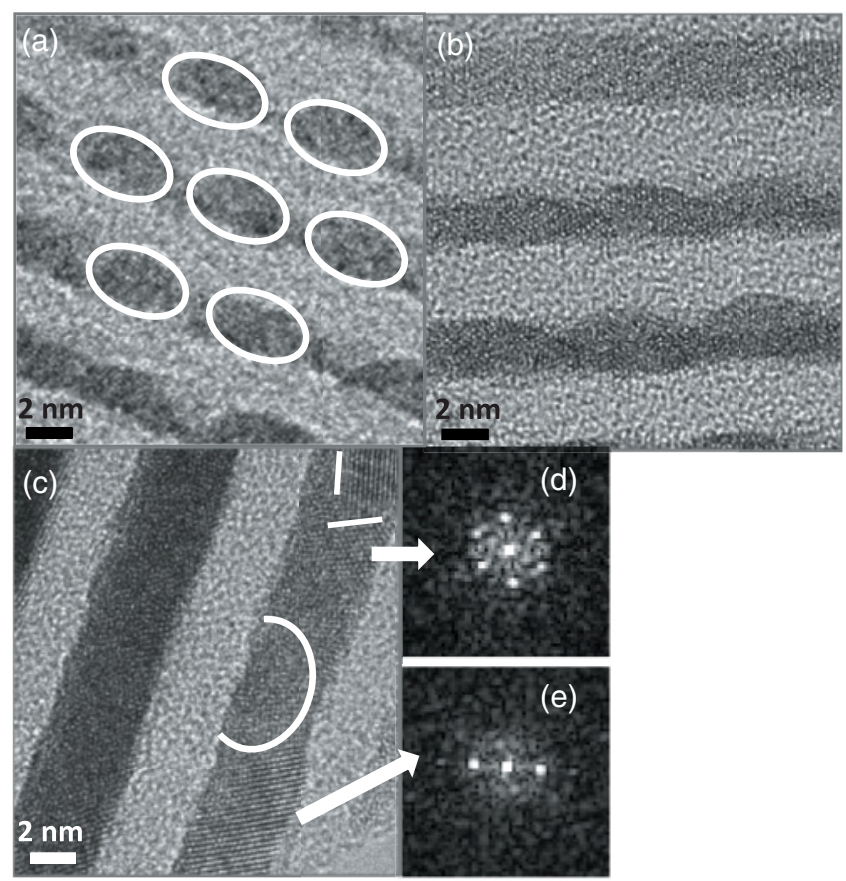

FIG. 1. HRTEM images in cross-section configuration for samples from the $a$ series with $t_{\mathrm{Co}}=0.7 \mathrm{~nm}$ and (a) $t_{\mathrm{W}}=0.6 \mathrm{~nm}$, (b) $t_{\mathrm{W}}=$ $1.5 \mathrm{~nm}$, and (c) $t_{\mathrm{W}}=4.5 \mathrm{~nm}$. Fourier transforms (FFTs) at selected zones of the image in (c) are shown in (d) and (e). Arrangement of the particles within layers is highlighted in (a). An amorphous zone on one of the layers of sample in (c) is demarcated by a white line. In (c), two white straight segments indicate two orientations of the (llll 110$)$ $\mathrm{W}$ planes.
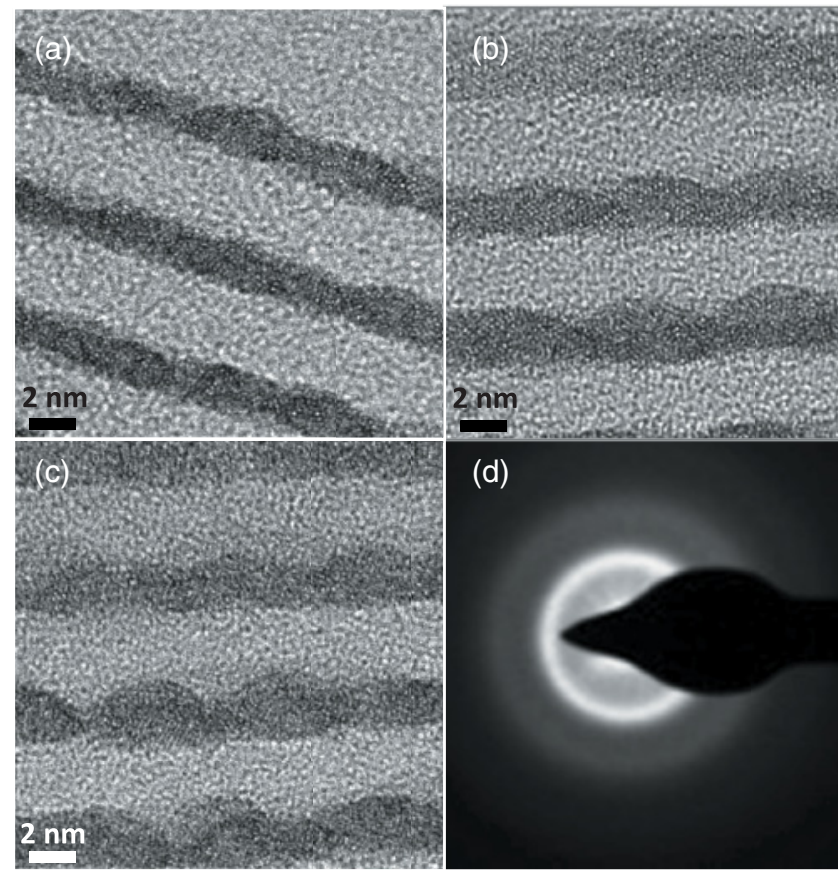

(d)

FIG. 2. HRTEM images in cross-section configuration for samples from the $b$ series with $t_{\mathrm{W}}=1.5 \mathrm{~nm}$ and (a) $t_{\mathrm{Co}}=0.4 \mathrm{~nm}$, (b) $t_{\mathrm{Co}}=0.7 \mathrm{~nm}$, and (c) $t_{\mathrm{Co}}=1.0 \mathrm{~nm}$. (d) SAED image of a NP in sample in (b).

being formed in our Co-W NPs. We will further discuss this topic in Sec. V.

The Co-W amorphous particles in series $a$ and $b$ are more detached in samples with higher $\mathrm{Co} / \mathrm{W}$ ratio, as in that of $t_{\mathrm{Co}}=$ $0.7 \mathrm{~nm}$ and $t_{\mathrm{W}}=0.6 \mathrm{~nm}$ [Fig. 1(a)]. In those samples where the $\mathrm{Co} / \mathrm{W}$ ratio decreases, $\mathrm{W}$ tends to connect the particles by filling the interparticle spaces [Figs. 1(b) and 1(c)]. These particles with elongated cross-sectional shape arrange within layers in a periodical way, similar to their assembly with alumina capping. ${ }^{39}$ That is, in each layer, particles lay on the depression formed by the previous deposited particles layer so that they form a hexagonal arrangement across the layers, as it is marked in Fig. 1(a). All this morphology described for samples from the $a$ series has also been observed for samples from the $b$ series (see Fig. 2).

Structural differences are found in the $a$-series sample with $t_{\mathrm{W}}=4.5 \mathrm{~nm}$. As it is observed in a HRTEM image for this sample [Fig. 1(c)], the interparticle spaces existing in all other four samples have been filled, so that the Co/W film has a nearly constant thickness. Each metallic layer of about $4.2 \mathrm{~nm}$ thick shows ordered zones alternating with amorphous zones. In the ordered regions, periodicity is around $0.2 \mathrm{~nm}$, confirmed by fast Fourier transform (FFT) performed on some of the ordered zones [see bright spots observed in Figs. 1(d) and 1(e)]. This periodicity and the distances and angles on the FFT are in agreement with the (lllllllanes of W bcc structure. There is also evidence of different grains within the crystalline zones on the layer, identified, for example, by the two orientations of the planes observed in the HRTEM image [see Fig. 1(c)], and the splitting of the spots in Fig. 1(d). EDS analysis shows the presence of both Co and W. However, it is not easy to establish differences between ordered and 


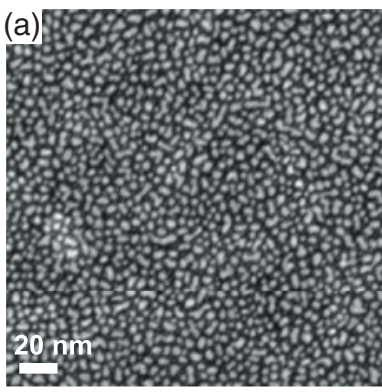

(c)
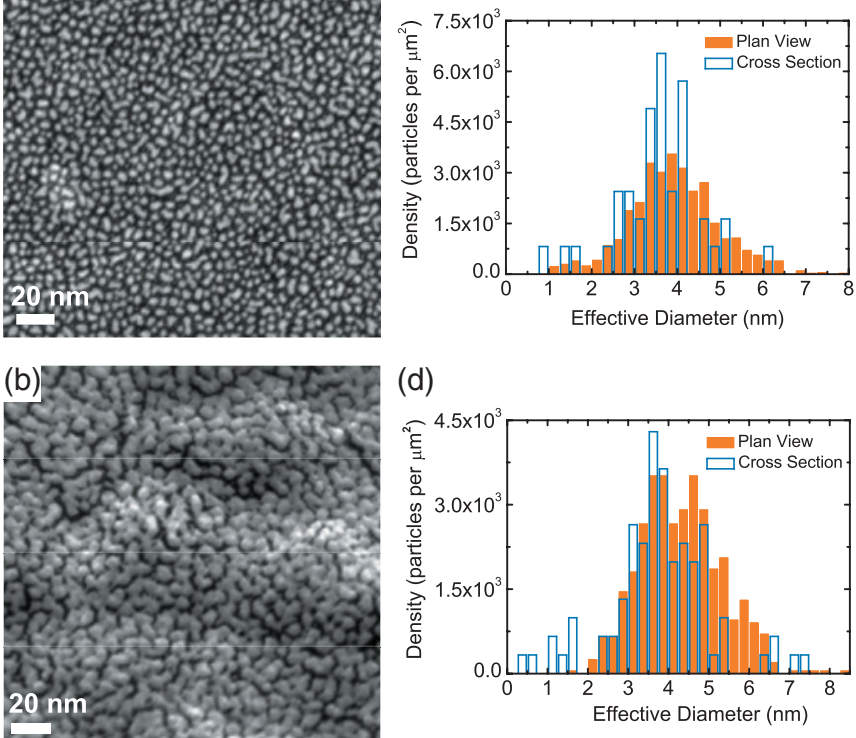

(d)

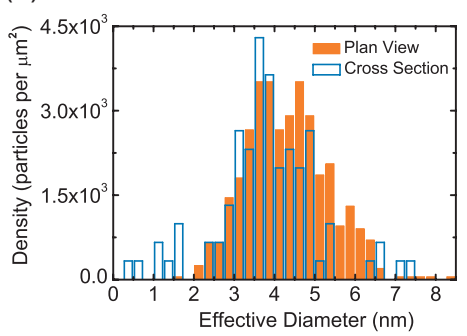

FIG. 3. (Color online) HAADF STEM images in plan-view configuration for the $a$ series samples with $t_{\mathrm{Co}}=0.7 \mathrm{~nm}$ and (a) $t_{\mathrm{W}}=0.6 \mathrm{~nm}$ and (b) $t_{\mathrm{W}}=1.5 \mathrm{~nm}$. Size distribution of plan-view and cross-section configurations for the samples in (a) and (b) are shown in (c) and (d), respectively.

disordered zones by this technique. Given the higher amount of $\mathrm{W}$ in this last sample, the $\mathrm{Co}-\mathrm{W}$ alloy is possibly saturated, so that $\mathrm{W}$ starts to generate the crystalline zones identified from the images [Fig. 1(c)]. Further discussion about $\mathrm{W}$ growing as a crystal in the samples is presented in the EXAFS section (see Sec. V).

High contrast obtained in plan-view HAADF STEM images for the $a$ series samples with $t_{\mathrm{Co}}=0.7 \mathrm{~nm}$ and $t_{\mathrm{W}}=0.6$ and $1.5 \mathrm{~nm}$ [Figs. 3(a) and 3(b)] allows easier identification of the particles than in HRTEM images due to the huge $Z$ contrast between the heavy metal particles and the light oxide matrix. In these images, brighter parts correspond to $\mathrm{Co}-\mathrm{W}$ nanoparticles and darker parts to $\mathrm{Al}_{2} \mathrm{O}_{3}$. Images obtained are comparable to those of similar systems of metallic nanoparticles embedded in insulator matrices, ${ }^{42}$ and the shape of the particles is similar to that of Co nanoparticles embedded in alumina. ${ }^{43}$ HAADF STEM images reveal that particles in the sample with $t_{\mathrm{W}}=$ $1.5 \mathrm{~nm}$ [Fig. 3(b)] are bigger and more closely packed than particles in the sample with $t_{\mathrm{W}}=0.6 \mathrm{~nm}$ [Fig. 3(a)].

Size distributions for samples from the $a$ series with $t_{\mathrm{Co}}=$ $0.7 \mathrm{~nm}$ and $t_{\mathrm{W}}=0.6 \mathrm{~nm}$ [Fig. 3(c)] and $1.5 \mathrm{~nm}$ [Fig. 3(d)] were obtained by digital processing of the images taken for both cross-section [Figs. 1(a) and 1(b)] and plan-view configurations [Figs. 3(a) and 3(b)]. Results for the diameter distribution on each case were fitted using a Gaussian distribution function. The values obtained from this analysis are listed in Table I. The effective diameters for each configuration, $\left\langle D_{\text {eff }}\right\rangle_{\mathrm{CS}}$ for cross section and $\left\langle D_{\text {eff }}\right\rangle_{\mathrm{PV}}$ for plan view, were obtained with the assumption that particles have circular in-plane and elliptic out-of-plane projection shapes, the latter with short axis $a$ and long axis $b$ on each projection, so that $D_{\text {eff }}=\sqrt{a b}$. Thus, Co-W particles have an oblate shape. Interparticle distance $d$ and surface density of particles $N_{S}$ expressed as the number of particles per $\mathrm{nm}^{2}$ for the plan-view images were also obtained (see Table I). These two values are almost equal for both samples analyzed in plan view, which means that particles are spatially distributed on each layer in the same way for those samples from the $a$ series. Cross-section values have a higher error since they were obtained from poor-contrast HRTEM images, so particle identification was less precise than in the HAADF STEM images in the plan-view case.

Digital analysis of the images obtained in cross section for samples from the $b$ series was also performed in order to identify some morphological parameters; however, size distributions in these cases were not obtained due to the low contrast and less separation between the particles in these two samples [see Figs. 2(a) and 2(c)]. A clear tendency in the size of the particles given by the $\mathrm{Co} / \mathrm{W}$ ratio in the samples is also evident from the values in Table I, so that $\left\langle D_{\text {eff }}\right\rangle_{\mathrm{CS}}$ increases from 2.84 to $4.24 \mathrm{~nm}$ for samples in the $b$ series, as $t_{\mathrm{Co}}$ increases from $0.4 \mathrm{~nm}$ to $1.0 \mathrm{~nm}$.

The elemental composition of the nanoparticles has been measured by EDS on the plan-view sample with $t_{\mathrm{Co}}=0.7 \mathrm{~nm}$ and $t_{\mathrm{W}}=0.6 \mathrm{~nm}$. A total of 12 particles were measured yielding to an average composition with tungsten content of $27 \pm 6$ at. $\% \mathrm{~W}$, i.e., the particle composition is close to that of $\mathrm{Co}_{3} \mathrm{~W}$. Moreover, the composition within one particle was constant within experimental error. The relative variation in the composition of the other particles has been determined by EXAFS analysis at the Co $K$ edge (see Sec. V).

From this morphological study, we conclude that we have obtained a system of self-organized and uniformly dispersed amorphous Co-W alloy NPs. This occurs since W has alloyed with the preformed Co NPs, instead of growing as a homogeneous crystalline film covering the particles. The size of the amorphous particles depends on the amount of $\mathrm{Co}$ and $\mathrm{W}$ deposited on each layer. Particles formed within the layers have an oblate shape. When the amount of $\mathrm{W}$ increases and the Co-W alloy saturates, $\mathrm{W}$ grows as a crystal and fills the interparticle spaces.

Previous works on $\mathrm{Co}-\mathrm{W}$ thin films have led to similar amorphous structure when such films are synthesized by sputtering, using composite targets. ${ }^{34,44}$ The amorphous forming ability (AFA) of $\mathrm{Co}-\mathrm{W}$ has been studied in terms of the Miedema's coordinates model $Y=\left|\Delta \phi / \Delta n_{\mathrm{WS}}^{1 / 3}\right|$, where $\Delta \phi=\phi_{\mathrm{Co}}-\phi_{\mathrm{W}}$, and $\Delta n_{\mathrm{WS}}=n_{\mathrm{WS}}(\mathrm{Co})-n_{\mathrm{WS}}(\mathrm{W})$, where $\phi$ is the chemical potential, and $n_{\mathrm{WS}}$ the electron density of the Wigner-Seitz unit cell, and $Z=\left|\left(R_{\mathrm{W}}-R_{\mathrm{Co}}\right) / R_{\mathrm{Co}}\right| .{ }^{44}$ The $\mathrm{Co}-\mathrm{X}(\mathrm{X}=\mathrm{Cr}, \mathrm{Mo}, \mathrm{W})$ binary alloy phase space is separated by the empirical law $Y=2.52 Z^{-1 / 4}$ in the amorphous and crystalline areas. It was found that $\mathrm{Co}-\mathrm{W}$ lies in the amorphous region; the reason for this favorable AFA in this alloy is a negative heat of mixing, large atomic size differences, and a low substrate temperature that avoids recrystallization via diffusion. These factors have explained satisfactorily the formation of $\mathrm{Co}-\mathrm{W}$ amorphous thin films by co-sputtering. ${ }^{34}$ In our preparation method, Co nanoparticles are formed first, and a subsequent $\mathrm{W}$ capping supplies the alloying metal. The sputtering voltage is enough to let $\mathrm{W}$ and Co react, while the substrate temperature avoids crystallization. The AFA is high since the $\mathrm{W}$ concentration is higher than $17 \%$, a condition needed for the formation enthalpy of the amorphous phase to be lower than the crystalline one. ${ }^{44}$ Therefore, amorphization 
TABLE I. Average particle diameter $\left(\left\langle D_{\text {eff }}\right\rangle\right)$, distribution width $(\sigma)$, interparticle distance $(d)$, and density of particles in the layer $\left(N_{S}\right)$ obtained from digital analysis of TEM images taken in cross-section (CS) and plan-view (PV) configurations.

\begin{tabular}{lccccccccc}
\hline \hline $\begin{array}{l}\text { Samples } \\
\text { series }\end{array}$ & $\begin{array}{c}t_{\mathrm{Co}} \\
(\mathrm{nm})\end{array}$ & $\begin{array}{c}t_{\mathrm{W}} \\
(\mathrm{nm})\end{array}$ & $\begin{array}{c}\left\langle D_{\mathrm{eff}}\right\rangle_{\mathrm{CS}} \\
(\mathrm{nm})\end{array}$ & $\begin{array}{c}\sigma_{\mathrm{CS}} \\
(\mathrm{nm})\end{array}$ & $\begin{array}{c}d_{\mathrm{CS}} \\
(\mathrm{nm})\end{array}$ & $\begin{array}{c}\left\langle D_{\text {eff }}\right\rangle_{\mathrm{PV}} \\
(\mathrm{nm})\end{array}$ & $\begin{array}{c}\sigma_{\mathrm{PV}} \\
(\mathrm{nm})\end{array}$ & $\begin{array}{c}d_{\mathrm{PV}} \\
(\mathrm{nm})\end{array}$ & $\begin{array}{c}N_{S} \\
\left(\times 10^{2} \mathrm{~nm}^{-2}\right)\end{array}$ \\
\hline$a$ & 0.7 & 0.6 & $3.6(1)$ & $0.4(2)$ & $5.8(5)$ & $3.87(4)$ & $0.82(6)$ & $5.3(5)$ & $3.2(1)$ \\
& 0.7 & 1.5 & $3.9(1)$ & $0.9(5)$ & $6.6(7)$ & $4.23(6)$ & $1.0(1)$ & $5.6(9)$ & $3.5(1)$ \\
$b$ & 0.4 & 1.5 & $2.84(2)$ & & $5.3(5)$ & & & & $3.6(9)$ \\
& 0.7 & 1.5 & $3.9(1)$ & $0.9(5)$ & $6.6(7)$ & $4.23(6)$ & $1.0(1)$ & $5.6(1)$ \\
& 1.0 & 1.5 & $4.24(2)$ & & $6.7(3)$ & & & & \\
\hline \hline
\end{tabular}

is favorable in the formation of Co-W NPs, just as it is in the thin-film case.

\section{STRUCTURAL STUDY: XANES AND EXAFS MEASUREMENTS}

The x-ray absorption spectra were analyzed following standard procedures. A preliminary reduction of the EXAFS raw data involving background removal of the absorption data $\mu(\mathrm{E})$, conversion of $\mu(\mathrm{E})$ to $\chi(\mathrm{k})$, normalization, and weighting scheme were performed with AUTOBK and ATHENA, programs from the simulation package IFEFFIT. ${ }^{45}$ EXAFS data analysis and fitting were performed on all reference and samples at their respective edges. All spectra were fitted using the ARTEMIS program from the same IFEFFIT package using models based on the crystallographic information found in the ICSD database. ${ }^{46}$ Atomic clusters used to generate the scattering paths for fitting were generated with ATOMS, a tool from the same simulation package. ${ }^{47}$

\section{A. Co $K$ edge}

X-ray absorption near edge structure spectra at Co $K$ edge for Co-W NPs from the $a$ and $b$ series are shown in Figs. 4(a) and 4(b), respectively. In both cases, a Co foil XANES is plotted for reference, and a spectrum for bare Co NPs with $t_{\text {Co }}=0.7 \mathrm{~nm}$ is plotted in Fig. 4(a) for comparison. Changes in the intensity of the pre-edge peak and the white line (denoted as peaks I and II, respectively, in Fig. 4) are noticeable. The variation of the peaks' areas $\left[\Delta \mathrm{A}=\int_{E_{i}}^{E_{f}}\left(\mu_{\text {sample }}-\mu_{\mathrm{Co}-\text { foil }}\right) d E\right]$ in series $a$ and $b$ is shown in the insets of Figs. 4(a) and 4(b), respectively. The integration limits were 7708.4-7721.3 eV for peak I and 7721.3-7729 eV for peak II.

The Co $K$ edge white line reflects its $4 p$ empty states near the Fermi energy. The origin of peak I is at the density of states (DOS) of $d$ character available at the Fermi energy, projected onto the Co site. ${ }^{48,49}$ This is influenced by intra-atomic Co $4 p-3 d$ hybridization. Moreover, in our samples, this $d$ DOS originates both at the Co $3 d$ band and at the $\mathrm{W} 5 d$ states through interatomic Co $3 d-\mathrm{W} 5 d$ hybridization. The intensity of peak I in Co-W NPs is clearly lower than in metal cobalt and lower than bare Co NPs for the $a$ samples series. The same reduction at peak I with respect to Co foil is observed for the $b$ series. Although alloying with $\mathrm{W}$ introduces a higher number of $d$ holes in the system, hybridization strongly delocalizes the $d$ empty states, depleting the intensity of peak I by reducing the $d$ DOS projected at the Co site. Therefore, the strong reduction of peak I at the Co $K$ edge of Co-W NPs is a proof of Co $4 p-3 d-\mathrm{W} 5 d$ hybridization.

In series $a$, peak I is practically constant along the series [see inset of Fig. 4(a)], reflecting that Co $3 d-\mathrm{W} 5 d$ hybridization is not affected by small variations in the amount of $\mathrm{W}$ for a fixed Co content. This, in principle, surprising fact strongly suggests that the alloy composition is similar for the three samples along
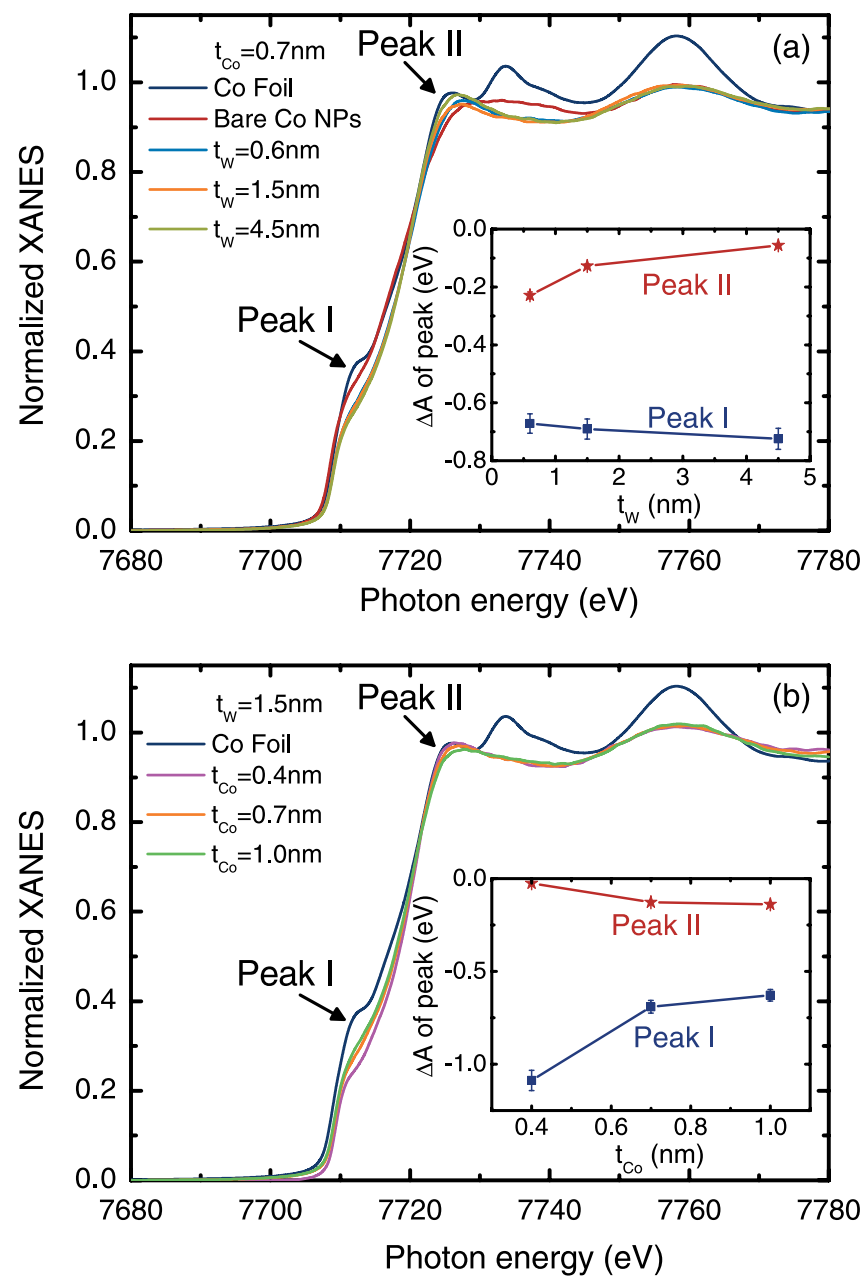

FIG. 4. (Color online) XANES at the Co $K$ edge for the Co-W NPs. (a) $a$ samples series. (b) $b$ samples series. A spectrum for the Co foil is plotted for reference in both cases. In (a), a curve for bare Co NPs with $t_{\mathrm{Co}}=0.7 \mathrm{~nm}$ has been included for comparison. Insets: Variation of the areas of peaks I and II in (a) and (b) as a function of $t_{\mathrm{W}}$ and $t_{\mathrm{Co}}$, respectively. 
this series, a result that will be confirmed by EXAFS (later in this section) and by magnetic analysis (Sec. VI). In contrast, $b$ samples series show a clear increase of the $\Delta \mathrm{A}$ of peak $\mathrm{I}$ upon increasing Co content in the NPs, as shown in the inset of Fig. 4(b). This trend is the expected one, as a decrease in the proportion of $\mathrm{W}$ (or increase of $\mathrm{Co} / \mathrm{W}$ ratio) in the sample lowers the number of $5 d$ holes available for hybridization, increasing the localization of the $d$-character states at the Co site.

The intensity of peak II depends on both the number of available $4 p$ states and the structure of the system in a nontrivial way. The comparison of the Co metal and Co-W NPs has to be taken with care, as their structures are different (hcp and amorphous, respectively). However, the trend of $\Delta \mathrm{A}$ of peak II in samples from the $a$ series indicates that the number of $4 p$ holes is enhanced as the $\mathrm{W}$ content increases, suggesting an electronic transfer from Co $4 p$ toward W $5 d$ states. Accordingly, $\Delta \mathrm{A}$ of peak II in samples from the $b$ series slightly decreases with Co content, suggesting that, for the range of $t_{\mathrm{Co}}$ and $t_{\mathrm{W}}$ studied, the number of $4 p$ holes is reduced as the ratio $\mathrm{Co} / \mathrm{W}$ increases. In short, along the $a$ and $b$ series, as the $\mathrm{Co} / \mathrm{W}$ ratio increases, both the $4 p$ number of empty states and the delocalization of $3 d$ empty states decrease.

Structural analysis that can be extracted from the $\chi(\mathrm{k})$ EXAFS signal is better studied by its Fourier transform, shown in Fig. 5, performed on a $k$ range from 2.0 to $11.9 \AA^{-1}$ using a $k^{2}$ weight and a hanning window function. This $R$-space data show peaks that correspond to different atomic shells surrounding the absorbing atom, Co in this case. In Fig. 5(a), it can be seen that the position and shape of the peaks in the bare Co nanoparticles and Co foil are very similar, not only for the first coordination shell, but also for outer shells, showing that cobalt in $\mathrm{Co} / \mathrm{Al}_{2} \mathrm{O}_{3}$ aggregates in a similar structure as metallic Co, as it has been reported in the literature. ${ }^{43}$ However, the peaks for outer coordination shells are different in the W-capped Co nanoparticles. This is caused by a change in the Co coordination due to alloying between Co-W, which is also evident in Fig. 5(b) for the $b$ sample series. Indeed, intensities of all peaks in both Figs. 5(a) and 5(b) are larger in the Co foil than in the particulate systems, as it is expected from the reduction in the mean Co coordination due to the size reduction.

In this study, the EXAFS signal for the bare Co nanoparticles with $t_{\mathrm{W}}=0.7 \mathrm{~nm}$ was fitted using a Co fcc metal model, where the coordination number $N$ was used as a fitting parameter. A reduction from $N=12$ in the metallic Co to $N=$ 6.1 in the first coordination shell of the bare Co nanoparticles was obtained from this fit. However, recent works have demonstrated that such a strong reduction in the number of first neighbors could be an artifact of EXAFS analysis, ${ }^{50}$ due to effects of surface distortions on the nanoparticles, producing an underestimation of the average coordination number.

A reduction in the EXAFS signal as a result of lower Co coordination due to alloying is also observed when the signal for the Co foil is compared with that for $\mathrm{Co}_{3} \mathrm{~W}$ in Figs. 5(a) and 5(b). This behavior supports the scheme of $\mathrm{Co}-\mathrm{W}$ alloy presence in the Co-W nanoparticles. There is a further reduction in the amplitude of all peaks and lack of structure beyond the first two coordination shells in the Co-W

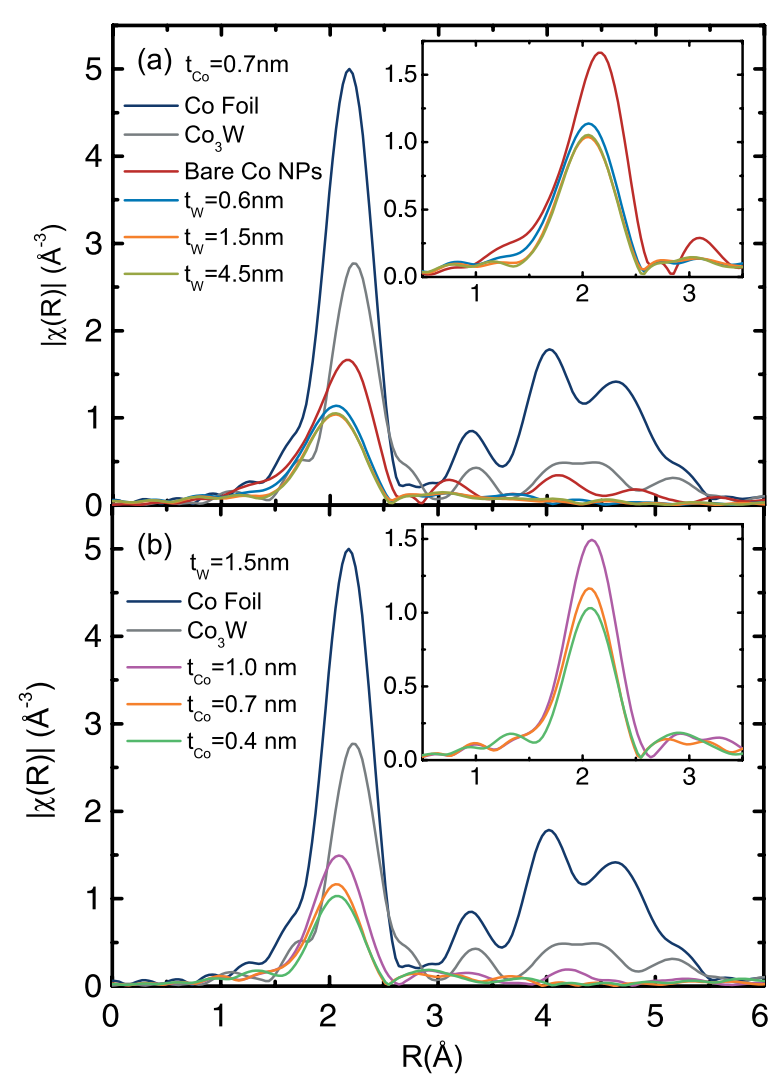

FIG. 5. (Color online) Fourier transform of EXAFS signal at the Co $K$ edge on the Co-W samples. (a) $a$ samples series. (b) $b$ samples series. Measurements for $\mathrm{Co}$ foil and $\mathrm{Co}_{3} \mathrm{~W}$ in both cases, and bare Co nanoparticles with $t_{\mathrm{Co}}=0.7 \mathrm{~nm}$ in (a) are shown for comparison. Inset: zoom of the first coordination shell in the particulate systems.

nanoparticles systems, which is characteristic of amorphicity, thus supporting the observation of amorphous structure in the HRTEM measurements. In general, amorphous compounds are characterized by damping of their EXAFS signal compared to a reference foil, consistent with static disorder and reduction of average coordination number. ${ }^{51}$

In the HRTEM section, we have mentioned that capping the Co nanoparticles with noble metals does not affect the structure of the NPs. ${ }^{14}$ This has also been observed with EXAFS, so that bare and Au-capped Co nanoparticles have equivalent fcc crystal structure when comparing their Fourier-transformed EXAFS signal. ${ }^{52}$ However, capping the Co NPs with W completely modifies the structure of the original particles by forming an amorphous alloy.

Different models were tested in order to fit the Co $K$ edge $R$-space data for the W-capped Co nanoparticles. Some of the fitting tests were performed by using a model of pure Co fcc metal, such as the one typically used in the bare Co nanoparticles case, and by using a $\mathrm{W}$-doped metal Co model. However, the best fitting model was obtained by using the $\mathrm{Co}$ scattering paths of the most stable $\mathrm{Co}-\mathrm{W}$ alloy, ${ }^{53}$ i.e., $\mathrm{Co}_{3} \mathrm{~W}$, and determining the deviation in the average coordination $(N)$ and interatomic distance $(r)$ for each sample. Given the high affinity of $\mathrm{Co}$ and $\mathrm{W}$ to form Co-W alloys, ${ }^{17,31-33}$ we have assumed that all Co in the sample is uniformly alloyed with W. Then, we have estimated the change of the Co environment 
when increasing the amount of $\mathrm{W}$ or Co in the sample, and the different trends the structural parameters follow when varying them.

Due to the amorphous nature of the Co-W NPs, it is very difficult to fit the EXAFS signal beyond the first coordination shell. Moreover, as it was mentioned above, strong structural disorder in the NPs systems can lead to erroneous interpretation of the EXAFS analysis. ${ }^{50,51}$ Then, fitting of the Co-W NPs EXAFS signal was performed on the $R$ space in a range from 1.0-3.0 $\AA$ using a hanning window function, so that it covered the Co first coordination shell in the alloy (see Fig. 6). According to the crystallographic information, this shell is composed by a combination of Co-Co and $\mathrm{Co}-\mathrm{W}$ paths (see Table II). The amplitude reduction factor $S_{0}^{2}$ and the shift in the threshold energy $\Delta E_{0}$ were set to those obtained for the Co foil fit. A total of 10 fitting parameters were used for an iterative procedure, which consistently included the fitting of both Co $K$ and W $L_{3}$ edges (see Sec. V B). Results for the Co-W NPs fits are shown in Fig. 6 and the values obtained in each case are listed in Table II.

A high accordance between the original EXAFS data and their fits is observed for all samples. Fitting results show that the interatomic distance $r$ and the disorder level quantified by the Debye-Waller factor $\sigma^{2}$ are strongly affected by the presence of $\mathrm{W}$ in the structure, showing the distortion of the $\mathrm{Co}-\mathrm{Co}$ and $\mathrm{Co}-\mathrm{W}$ bonds in the amorphous Co-W alloy. These values found for $\sigma^{2}$ are typical of disordered Co environments,

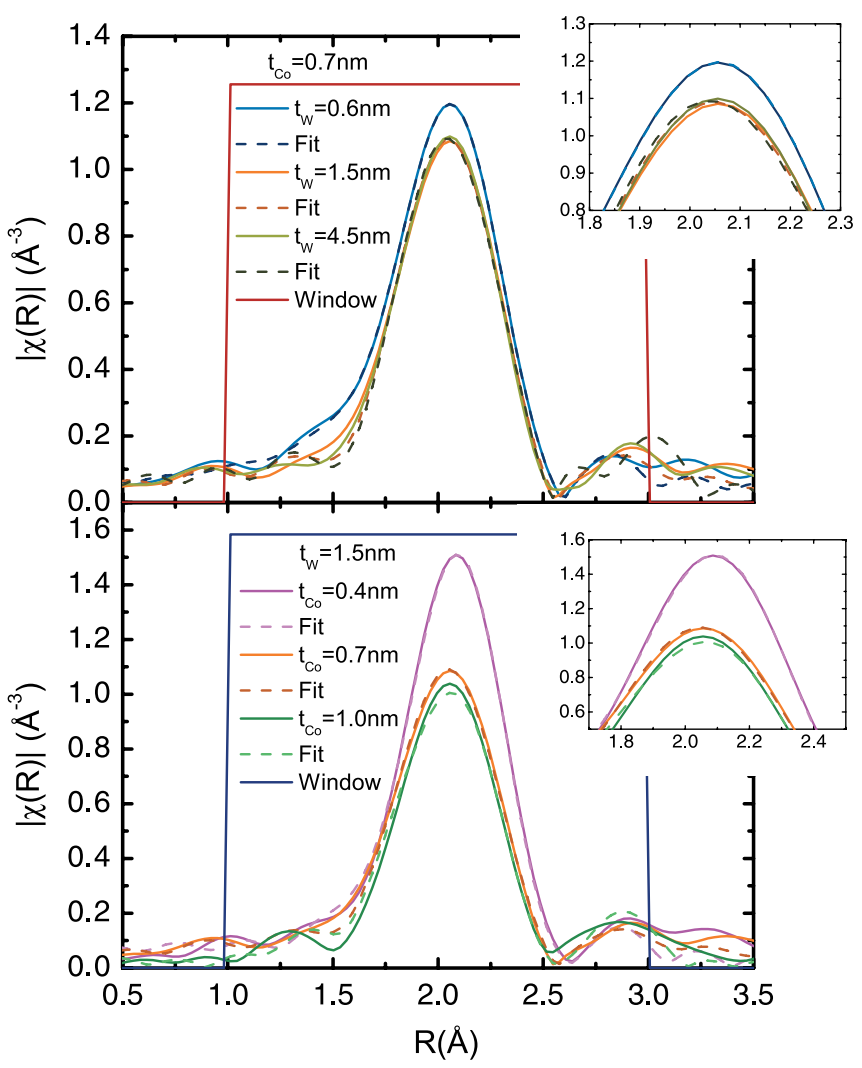

FIG. 6. (Color online) Best fits to the first coordination shell on the Co $K$ edge $R$-space EXAFS signal for all five Co-W nanoparticles samples studied. (a) $a$ samples series. (b) $b$ samples series. Inset: zoom of the fitted peak. e.g., in Co-rich amorphous alloys. ${ }^{54}$ It is also observed that the highest $\sigma^{2}$ is obtained in the Co-W bonds, confirming the high amorphicity created by $\mathrm{W}$ in the Co nanoparticles.

The average total coordination of the central Co atom $N_{\mathrm{T}}$, which includes both $\mathrm{Co}-\mathrm{Co}$ and $\mathrm{Co}-\mathrm{W}$ coordination, is reduced from $N=12$ in the $\mathrm{Co}_{3} \mathrm{~W}$ alloy to values as low as $N=9.4$, which corresponds to a decrease of about $22 \%$. This reduction is observed as the amount of $\mathrm{W}$ in the sample increases in the $a$ series and the amount of Co decreases in the $b$ series. However, $N_{\mathrm{T}}$ in most samples still resembles that of the crystal. This is an indication of short-range ordering in our samples, of chemical or structural origin, or both. Such SRO has been detected in similar $T_{1-x} M_{x}$ alloys ( $T$ a transition metal and $M$ a metal that favors amorphicity) via EXAFS and electron diffraction. ${ }^{41,55}$ In these alloys, it was found that SRO increases with increasing $T$ content. Indeed, we identify a similar trend of $N_{\mathrm{T}}$ approaching the $N=12$ value in our Co-W NPs as the amount of Co increases in the sample, suggesting a higher $\mathrm{SRO}$ in the sample with $t_{\mathrm{Co}}=1.0 \mathrm{~nm}$.

Variation of the coordination of Co atoms in the Co-W NPs samples with $t_{\mathrm{Co}}$ and $t_{\mathrm{W}}$ is plotted in Fig. 7 . When analyzing only the coordination between the central Co and their first Co neighbors $N_{\text {Co-Co }}$ [left $y$ axis in Figs. 7 (a) and 7(b)], a clear decrease from $N_{\mathrm{Co}-\mathrm{Co}}=8$ in $\mathrm{Co}_{3} \mathrm{~W}$ to values as low as $N_{\text {Co-Co }}=4.6$ for the smallest particles, is observed. This is a reduction of about $42 \%$ in the Co-Co coordination. In general, it is found that the $N_{\mathrm{Co}-\mathrm{Co}}$ decreases as the amount of Co decreases and the $\mathrm{W}$ content increases in the $b$ and $a$ samples series, respectively. Similarly, the average coordination of $\mathrm{W}$ atoms surrounding the central $\mathrm{Co}\left(N_{\mathrm{Co}-\mathrm{W}}\right)$ increases as the amount of $\mathrm{W}$ in the sample increases, and as the amount of $\mathrm{Co}$ decreases, following an almost linear dependence with $t_{\mathrm{Co}}$ and $t_{\mathrm{W}}$ [see right $y$ axis in Figs. 7(a) and 7(b)].

A slightly higher coordination has been observed for the sample with the lowest amount of Co $\left(t_{\mathrm{Co}}=0.4 \mathrm{~nm}\right.$ and $t_{\mathrm{W}}=$ $1.5 \mathrm{~nm})$, which seems to deviate from the trend given the small size of the particles in this sample. This behavior is probably due to the high $x_{\text {para }}$ present in this case $\left(x_{\text {para }}=0.5\right)$ so that the system is less particulate than the others, forming an almost uniformly distributed Co-W amorphous alloy on each layer.

\section{B. W $L_{3}$ edge}

XANES spectra at the $\mathrm{W} L_{3}$ edge for Co-W NPs are shown in Figs. 8(a) and 8(b) for $a$ and $b$ sample series, respectively. XANES of $\mathrm{W}$ foil at the same edge is plotted in both cases for comparison. In the case of $\mathrm{W}$, being a $5 d$ metal, $L_{3}$ absorption edge appears for transitions from $2 p$ to both $5 d_{3 / 2}$ and $5 d_{5 / 2}$ bands. Then, an increase in $\mathrm{W} L_{3}$ edge intensity from one sample to the other shows an increase in the number of $\mathrm{W}$ $5 d$ available empty states. In the $a$ series samples [Fig. 8(a)], intensity decreases as $t_{\mathrm{W}}$ increases. For the $b$ series [Fig. 8(b)], the white line is systematically enhanced as the amount of $\mathrm{Co}$ increases from zero, in the case of $\mathrm{W}$ foil, to $t_{\mathrm{Co}}=1.0 \mathrm{~nm}$. In both cases, an increase in the $\mathrm{Co} / \mathrm{W}$ ratio gives rise to an increase in the number of W $5 d$ empty states. We may conclude that, as this $\mathrm{Co} / \mathrm{W}$ ratio increases, there is an electron transfer from $\mathrm{W}$ toward Co. 
TABLE II. Structural parameters obtained from the fits for the Co-W nanoparticles shown in Fig. 6 and comparison with the nominal values for the $\mathrm{Co}_{3} \mathrm{~W}$ alloy from ICSD database (Ref. 46). Coordination numbers $N$, interatomic distance $r$, and Debye-Waller factor $\sigma^{2}$ for each path used in the fitting model.

\begin{tabular}{|c|c|c|c|c|c|c|c|c|c|c|c|}
\hline \multicolumn{3}{|c|}{$\mathrm{Co}_{3} \mathrm{~W}$ alloy } & \multicolumn{9}{|c|}{$a$ samples series $\left(t_{\mathrm{Co}}=0.7 \mathrm{~nm}\right)$} \\
\hline \multirow[b]{2}{*}{ Bond } & \multirow[b]{2}{*}{$N$} & \multirow[b]{2}{*}{$r(\AA)$} & \multicolumn{3}{|c|}{$t_{\mathrm{W}}=0.6 \mathrm{~nm}$} & \multicolumn{3}{|c|}{$t_{\mathrm{W}}=1.5 \mathrm{~nm}$} & \multicolumn{3}{|c|}{$t_{\mathrm{W}}=4.5 \mathrm{~nm}$} \\
\hline & & & $N$ & $r(\AA)$ & $\sigma^{2}\left(\AA^{2}\right)$ & $N$ & $r(\AA)$ & $\sigma^{2}\left(\AA^{2}\right)$ & $N$ & $r(\AA)$ & $\sigma^{2}\left(\AA^{2}\right)$ \\
\hline $\mathrm{Co}-\mathrm{Co}$ & 2.0 & 2.401 & $2.0(2)$ & $2.33(2)$ & $0.004(2)$ & $2.0(8)$ & $2.37(4)$ & $0.0040(4)$ & $2.0(8)$ & $2.36(1)$ & $0.0020(3)$ \\
\hline $\mathrm{Co}-\mathrm{Co}$ & 4.0 & 2.486 & $1.8(3)$ & $2.46(2)$ & $0.002(2)$ & $1.8(5)$ & $2.51(5)$ & $0.0044(7)$ & $1.8(1)$ & $2.52(1)$ & $0.0014(3)$ \\
\hline Co-W & 2.0 & 2.567 & $1.7(2)$ & $2.19(2)$ & $0.024(6)$ & $1.5(6)$ & $2.14(9)$ & $0.07(1)$ & $2.7(5)$ & $2.22(1)$ & $0.032(3)$ \\
\hline Co-W & 2.0 & 2.597 & $2.5(2)$ & $2.61(2)$ & $0.009(2)$ & $3.0(8)$ & 2.63(9) & $0.015(2)$ & $3.0(3)$ & $2.45(1)$ & $0.010(1)$ \\
\hline $\mathrm{Co}-\mathrm{Co}$ & 2.0 & 2.729 & $3.6(2)$ & $2.61(2)$ & $0.018(4)$ & $1.1(7)$ & $2.71(8)$ & $0.012(3)$ & $0.8(5)$ & $3.36(1)$ & $0.006(2)$ \\
\hline$N_{\mathrm{T}}$ & 12 & & $11.5(3)$ & & & $9.4(8)$ & & & $10.3(8)$ & & \\
\hline$N_{\text {Co-Co }}$ & 8 & & $7.4(3)$ & & & $4.9(8)$ & & & $4.6(8)$ & & \\
\hline$N_{\mathrm{Co}-\mathrm{W}}$ & 4 & & $4.1(3)$ & & & $4.5(8)$ & & & $5.7(8)$ & & \\
\hline \multicolumn{3}{|c|}{$\mathrm{Co}_{3} \mathrm{~W}$ alloy } & \multicolumn{9}{|c|}{$b$ samples series $\left(t_{\mathrm{W}}=1.5 \mathrm{~nm}\right)$} \\
\hline & & & \multicolumn{3}{|c|}{$t_{\mathrm{Co}}=0.4 \mathrm{~nm}$} & \multicolumn{3}{|c|}{$t_{\mathrm{Co}}=0.7 \mathrm{~nm}$} & \multicolumn{3}{|c|}{$t_{\mathrm{Co}}=1.0 \mathrm{~nm}$} \\
\hline Bond & $N$ & $r(\AA)$ & $N$ & $r(\AA)$ & $\sigma^{2}\left(\AA^{2}\right)$ & $N$ & $r(\AA)$ & $\sigma^{2}\left(\AA^{2}\right)$ & $N$ & $r(\AA)$ & $\sigma^{2}\left(\AA^{2}\right)$ \\
\hline Co-Co & 2.0 & 2.401 & $1.8(3)$ & $2.27(2)$ & $0.0046(8)$ & $2.0(8)$ & $2.37(4)$ & $0.0040(4)$ & $2.0(4)$ & $2.32(1)$ & $0.0031(3)$ \\
\hline $\mathrm{Co}-\mathrm{Co}$ & 4.0 & 2.486 & $1.8(1)$ & $2.41(2)$ & $0.0033(6)$ & $1.8(5)$ & $2.51(5)$ & $0.0044(7)$ & $4.0(6)$ & $2.48(1)$ & $0.0047(2)$ \\
\hline Co-W & 2.0 & 2.567 & $3.5(6)$ & $2.21(3)$ & $0.014(2)$ & $1.5(6)$ & $2.14(9)$ & $0.07(1)$ & $2.0(4)$ & $2.21(2)$ & $0.012(1)$ \\
\hline Co-W & 2.0 & 2.597 & $3.0(8)$ & $3.02(8)$ & $0.021(4)$ & $3.0(8)$ & $2.63(9)$ & $0.015(2)$ & $2.0(6)$ & $2.40(2)$ & $0.0038(3)$ \\
\hline $\mathrm{Co}-\mathrm{Co}$ & 2.0 & 2.729 & $1.0(8)$ & $3.23(7)$ & $0.011(3)$ & $1.1(7)$ & $2.71(8)$ & $0.012(3)$ & $2.0(6)$ & $2.82(2)$ & $0.013(1)$ \\
\hline$N_{\mathrm{T}}$ & 12 & & $11.1(8)$ & & & $9.4(8)$ & & & $12.0(6)$ & & \\
\hline$N_{\text {Co-Co }}$ & 8 & & $4.6(8)$ & & & $4.9(8)$ & & & $8.0(6)$ & & \\
\hline$N_{\mathrm{Co}-\mathrm{W}}$ & 4 & & $6.5(8)$ & & & $4.5(8)$ & & & $4.0(6)$ & & \\
\hline
\end{tabular}

Fourier transform of the EXAFS data taken at the $\mathrm{W} L_{3}$ edge for the Co-NPs is shown in Fig. 9. Fourier transformation was performed on the $\chi(\mathrm{k})$ EXAFS signal on a $k$ range from 2.3 to $9.8 \AA^{-1}$ using a $k^{2}$ weight and a sine window function. Figure 9(a) shows results for samples from the $a$ series and Fig. 9(b)
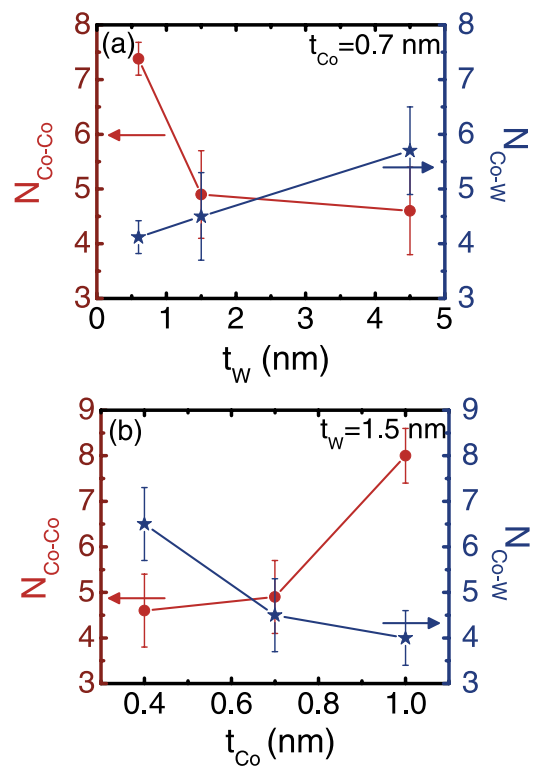

FIG. 7. (Color online) Variation of $N_{\mathrm{Co}-\mathrm{Co}}$ and $N_{\mathrm{Co}-\mathrm{W}}$ obtained from EXAFS fits. (a) $a$ samples series. (b) $b$ samples series. for the $b$ series, as well as for a $\mathrm{W}$ foil used as a reference and for $\mathrm{Co}_{3} \mathrm{~W}$ alloy for comparison. In both cases, it is evident that the $\mathrm{W}$ neighborhood has changed in the $\mathrm{Co}-\mathrm{W}$ nanoparticles compared to that of metallic $\mathrm{W}$ by the presence of $\mathrm{Co}-\mathrm{W}$ alloying. The first peak in the $\mathrm{Co}-\mathrm{W}$ samples is very close to that of $\mathrm{Co}-\mathrm{W}$ in the $\mathrm{Co}_{3} \mathrm{~W}$ alloy. For the sample with $t_{\mathrm{W}}=0.6 \mathrm{~nm}$ and $t_{\mathrm{Co}}=0.7 \mathrm{~nm}$, this peak amplitude is higher, showing a higher amount of Co neighbors in the $\mathrm{W}$ environment.

EXAFS spectra at the W $L_{3}$ edge were fitted following a similar procedure to the one for Co $K$ edge, which included an

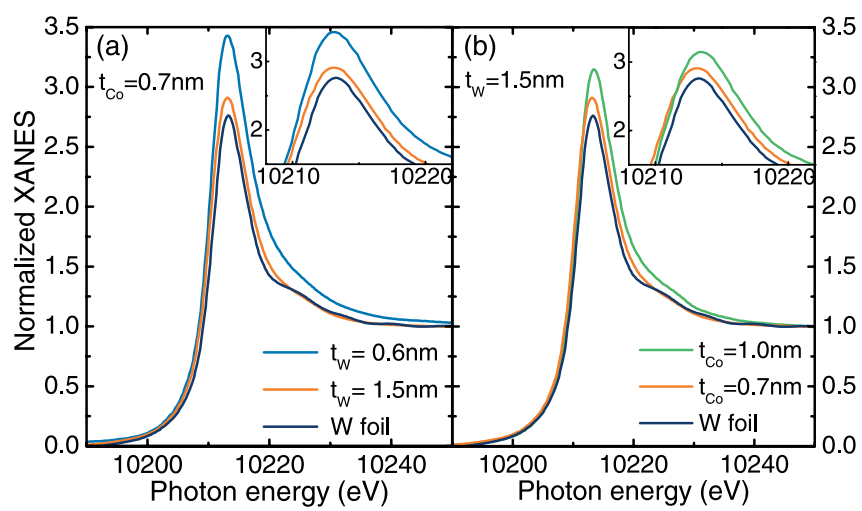

FIG. 8. (Color online) XANES spectra at the W $L_{3}$ edge for samples from the (a) $a$ samples series and (b) $b$ samples series. In both cases, the absorption of the $\mathrm{W}$ foil is shown for reference. Insets show details of the white-line intensity. 


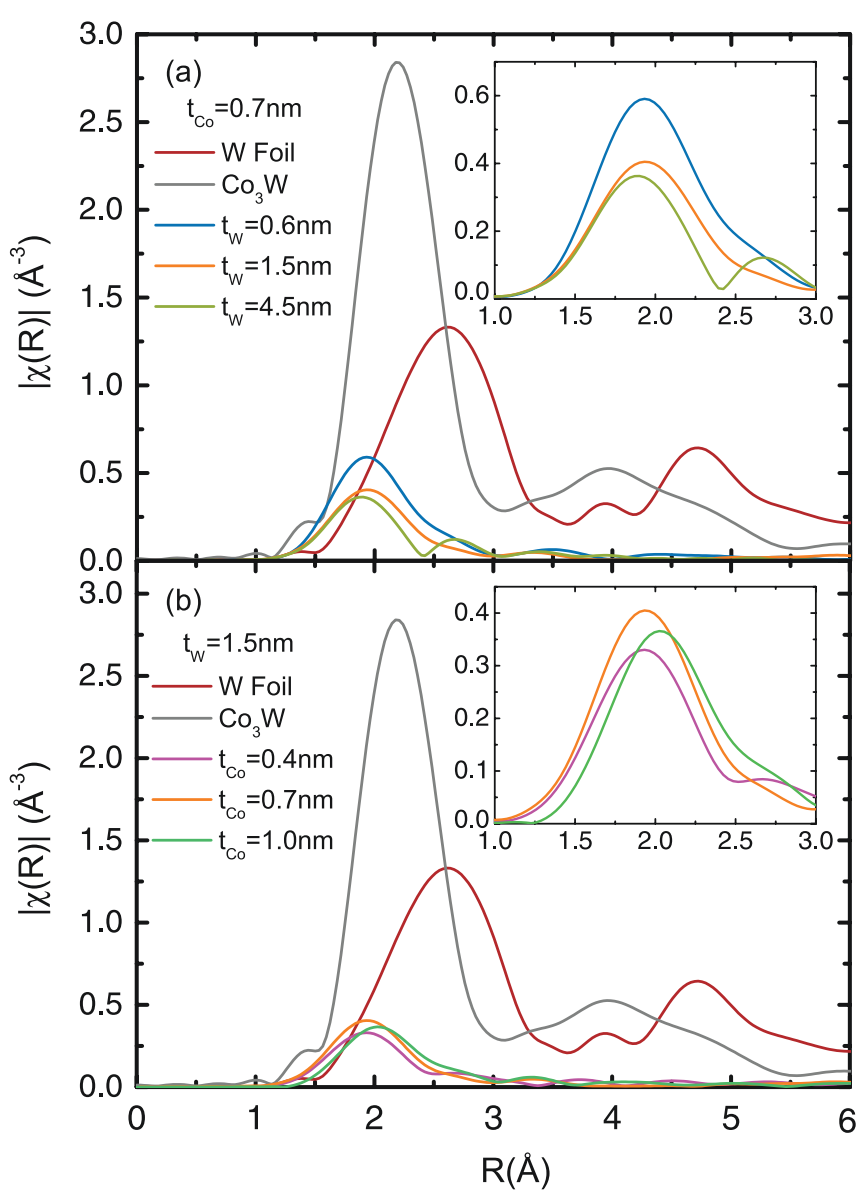

FIG. 9. (Color online) Fourier transform of EXAFS signal at the W $L_{3}$ edge on the Co-W NPs studied. (a) $a$ samples series. (b) $b$ samples series. Measurements for $\mathrm{W}$ foil and $\mathrm{Co}_{3} \mathrm{~W}$ are shown for comparison in both graphs. Insets: zoom of the first coordination shell in the particulate systems.

iterative fitting process of both edges. Fitting was performed on the $R$ space in a range that covered the $\mathrm{W}$ first coordination shell (1.5-3.0 $\AA$ ), using a hanning window function (see Fig. 10). $S_{0}^{2}$ and $\Delta E_{0}$ were set to those values obtained for the $\mathrm{W}$ foil signal fit. Different models for fitting were tested: a pure metallic $\mathrm{W}$ model, a pure $\mathrm{Co}-\mathrm{W}$ alloy $\left(\mathrm{Co}_{3} \mathrm{~W}\right)$, and a linear combination of these two phases. The latter was the procedure leading to the best fitting results, shown in Fig. 10.

The $\mathrm{W}$ environment was found to be consistent with the results of Co-W alloy obtained for the Co $K$ edge, showing evidence of an additional $\mathrm{W}$ metal phase in all samples. However, a quantitative determination of this $\mathrm{W}$ metal fraction was prevented given the intrinsic difficulties of fitting EXAFS signals for amorphous systems, as mentioned above. ${ }^{50,51} \mathrm{In}$ spite of it, $\mathrm{W} L_{3}$ EXAFS results show interesting trends as shown in Fig 10(a), where it is noticeable that the shoulder corresponding to the metallic $\mathrm{W}$ contribution increases as the amount of $\mathrm{W}$ increases in the sample ( $a$ series). A similar trend was also observed in their fitting results and is well in agreement to what was observed by HRTEM. The fraction of $\mathrm{W}$ metal was found to be around an almost constant value when fitting the samples from the $b$ series [Fig. 10(b)].

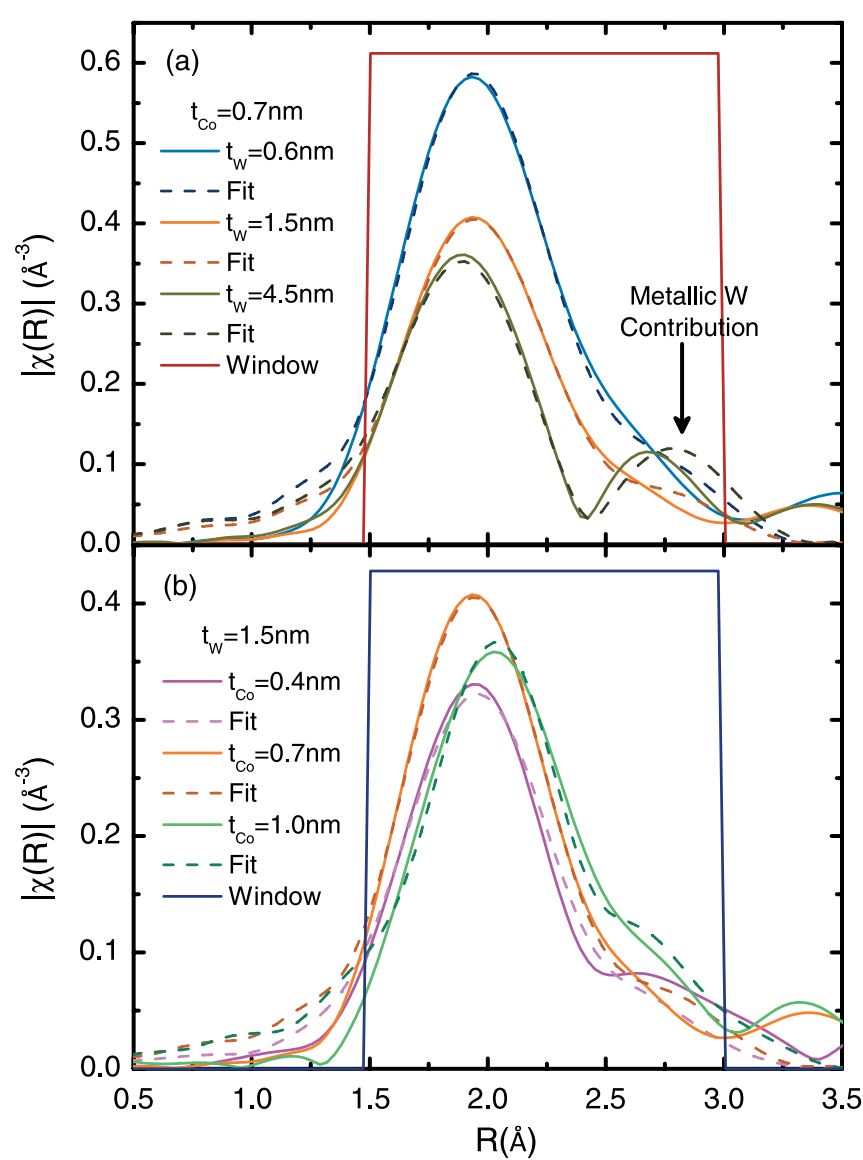

FIG. 10. (Color online) Best fits to the first coordination shell on $R$-space EXAFS signal measured at the $\mathrm{W} L_{3}$ edge for the Co-W nanoparticles samples. (a) $a$ samples series. (b) $b$ samples series.

\section{MAGNETIC STUDY: SQUID-BASED MAGNETOMETRY}

\section{A. Superparamagnetic Co-W particles}

SQUID-based magnetic measurements reveal the superparamagnetic behavior of the amorphous Co-W NPs. ZFC and FC curves for both sample series (Fig. 11) are similar to those for bare and $\mathrm{Cu}, \mathrm{Ag}$, and Au-capped Co nanoparticles. ${ }^{10,11,14}$ At lower temperatures, FC and ZFC for each sample separate, and the ZFC curve shows a maximum at a blocking temperature $T_{B}$. The values of $T_{B}$ for the Co-W NPs are given in Table III. $T_{B}$ values in all samples are lower than those found for the bare Co-NPs. ${ }^{10} T_{B}$ is also reduced for the $a$ series as the amount of $\mathrm{W}$ in the sample increases for a constant $t_{\mathrm{Co}}$ [Fig. 11(a)] and for samples from the $b$ series when the amount of Co decreases at a fixed $t_{\mathrm{W}}$ [Fig. 11(b)]. At high temperatures, all curves follow the Curie-Weiss law $\chi_{\mathrm{eq}}=C /(T-\theta)$. The values of the Curie-Weiss temperature $\theta$ obtained from the curves are shown in Table III.

Although $\theta$ values are not negligible, some trends are identified in order to describe the magnetic interactions between Co-W NPs. For the $a$ series samples, $\theta$ remains approximately constant as the amount of $\mathrm{W}$ increases, suggesting that interparticle interactions are quite similar in the series and decrease on a small scale when adding $\mathrm{W}$. This is understandable since particles are similarly and uniformly distributed on the layers 


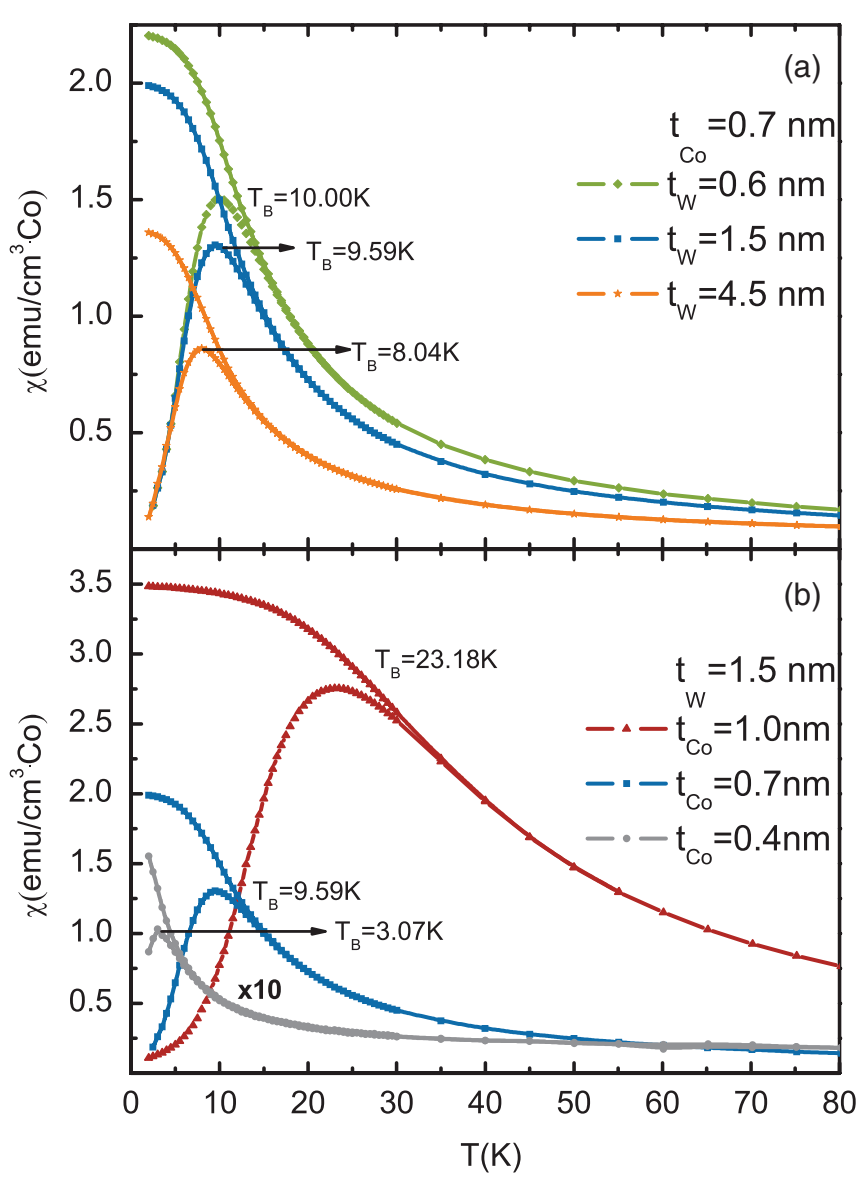

FIG. 11. (Color online) dc susceptibility curves for samples from the $a$ series in (a) and from the $b$ series in (b).

of these three samples, as demonstrated by HRTEM (see analysis of $N_{S}$ in Sec. IV). Instead, for the $b$ series, $\theta$ values have a drastic change, increasing in one order of magnitude from sample with $t_{\mathrm{Co}}=0.4 \mathrm{~nm}$ to the one with $t_{\mathrm{Co}}=1.0$ $\mathrm{nm}$. Thus, the magnetic interactions in the Co-W NPs increase as the amount of Co increases because particles are bigger and closer. These results of the magnetic interactions in the Co-W NPs are well supported by analysis of the variation of the FC-ZFC curves' height ratios, ${ }^{56}$ which show the same trend. That is, FC-ZFC curves' height ratios for the $a$ series are almost equal and they decrease as $t_{\mathrm{Co}}$ increases for the $b$ series samples.

Magnetization as a function of field in the paramagnetic region $\left[M(H)\right.$ at $\left.T \approx 3 T_{B}\right]$ for both sample series are plotted in Fig. 12. These $M(H)$ curves were identical when measured with the field parallel and perpendicular to the sample plate, therefore, the particle anisotropy axes are randomly distributed in all samples and the demagnetization factor plays no role in the present case.

Values of the magnetization per Co atom deposited in the samples $m_{\text {at }}$ obtained from saturation are listed in Table III and plotted in Fig. 13. It was found that $m_{\text {at }}$ decreases for samples from $a$ series as the $\mathrm{W}$-capping layer thickness increases for a constant $t_{\text {Co }}$ [Figs. 12(a) and 13(a)] and in samples from the $b$ series as the amount of Co decreases for a fixed $t_{\mathrm{W}}$ [Figs. 12(b) and 13(b)]. The magnetization is supported by the Co atoms since $\mathrm{W}$ has a negligible induced moment. ${ }^{57}$ Then, there is a strong reduction on the magnetic moment of the Co atoms due to the formation of the $\mathrm{Co}-\mathrm{W}$ amorphous alloy. This reduction may be due to combined effects of charge transfer, hybridization, and environment, as it is described in Ref. 31. According to this author, when the number of Co first neighbors is higher than 3 , Co atoms carry their full magnetic moment of $1.7 \mu_{B} / \mathrm{Co}^{31}$ This is the case of previously studied bare Co nanoparticles. ${ }^{11}$ However, if the $\mathrm{Co}$ atom has less $\mathrm{Co}$ atoms as first neighbors, e.g., 2 or 3 Co atoms, its magnetic moment decreases to about 0.5 $\mu_{B} / \mathrm{Co}$. The latter value is well in agreement with the moments found for Co atoms in the samples studied in this work. The strong reduction of the Co moment in our samples is due to a decrease in the Co coordination of the Co atoms because of the presence of $\mathrm{W}$, demonstrated via EXAFS analysis in the previous section. Such a decrease in $T_{1-x} M_{x}$ alloys ( $T$ being a transition metal and $M$ usually a nonmagnetic metal) has been reported in previous works ${ }^{58}$ and will be further discussed in Sec. VIII.

For those samples where the number of particles per unit surface $N_{S}$ was identified from the STEM images in the morphology characterization, we can easily determine the magnetic moment per particle $m_{\text {Part }}$. This is obtained by dividing the saturation magnetization by $N_{S}$, both per unit surface. $m_{\text {Part }}$ values are listed in Table III. The average amount of Co atoms per particle is also easily determined by the ratio of deposited $\mathrm{Co}$ and number of particles, per unit surface. The resulting moment per Co atom (Table III) decreases with increasing the amount of $\mathrm{W}$ in the $a$ series samples from 0.52 to $0.30 \mu_{B} / \mathrm{Co}$, for $t_{\mathrm{W}}=0.6$ and $4.5 \mathrm{~nm}$, respectively. Also, it strongly decreases with reduction of the amount of Co in the $b$ series samples from 0.93 to $0.08 \mu_{B} /$ Co for $t_{\mathrm{Co}}=1.0$ and 0.4 $\mathrm{nm}$, respectively. Thus, the reduction in Co moment expected for Co-W alloying ${ }^{17,31}$ is properly verified.

TABLE III. Summary of the parameters deduced from the magnetic measurements on Co-W nanoparticles.

\begin{tabular}{lcccccccccccc}
\hline \hline $\begin{array}{l}\text { Samples } \\
\text { series }\end{array}$ & $\begin{array}{c}t_{\mathrm{Co}} \\
(\mathrm{nm})\end{array}$ & $\begin{array}{c}t_{\mathrm{W}} \\
(\mathrm{nm})\end{array}$ & $\begin{array}{c}T_{B} \\
(\mathrm{~K})\end{array}$ & $\begin{array}{c}\theta \\
(\mathrm{K})\end{array}$ & $\begin{array}{c}m_{\mathrm{at}} \\
\left(\mu_{B} / \mathrm{Co}\right)\end{array}$ & $\begin{array}{c}m_{\text {Part }} \\
\left(10^{3} \mu_{B}\right)\end{array}$ & $\begin{array}{c}x \text { in Co-W } \\
\text { alloy }(\%)\end{array}$ & $\begin{array}{c}M_{\mathrm{sb}} \\
\left(10^{3} \mathrm{emu} / \mathrm{cm}^{3}\right)\end{array}$ & $\begin{array}{c}\left\langle D_{\text {mag }}\right\rangle \\
(\mathrm{nm})\end{array}$ & $\begin{array}{c}\sigma_{\text {mag }} \\
(\mathrm{nm})\end{array}$ & $\begin{array}{c}\langle U\rangle \\
(\mathrm{K})\end{array}$ & $\begin{array}{c}K_{\mathrm{eff}} \\
\left(10^{6} \mathrm{erg} / \mathrm{cm}^{3}\right)\end{array}$ \\
\hline$a$ & 0.7 & 0.6 & $10.00(6)$ & $7.3(2)$ & $0.52(2)$ & $1.03(1)$ & $18.2(4)$ & $0.413(2)$ & $3.4(2)$ & $0.25(5)$ & $270(10)$ & $1.4(3)$ \\
& 0.7 & 1.5 & $9.59(6)$ & $8.0(3)$ & $0.46(1)$ & $0.84(1)$ & $18.8(4)$ & $0.367(2)$ & $3.2(2)$ & $0.32(5)$ & $220(4)$ & $1.2(2)$ \\
& 0.7 & 4.5 & $8.04(3)$ & $5.9(2)$ & $0.30(1)$ & $0.57(1)$ & $20.3(4)$ & $0.235(2)$ & $3.4(6)$ & $0.38(5)$ & $200(10)$ & $1.0(5)$ \\
$b$ & 0.4 & 1.5 & $3.07(6)$ & $1.7(9)$ & $0.088(2)$ & & $22.3(4)$ & $0.062(2)$ & $2.6(5)$ & $0.25(5)$ & \\
& 0.7 & 1.5 & $9.59(6)$ & $8.0(3)$ & $0.46(1)$ & $0.84(1)$ & $18.8(4)$ & $0.367(2)$ & $3.2(2)$ & $0.32(5)$ & $220(4)$ & $1.2(2)$ \\
& 1.0 & 1.5 & $23.18(7)$ & $13.3(9)$ & $0.93(3)$ & & $14.1(4)$ & $0.725(2)$ & $4.4(2)$ & $0.38(5)$ & $890(30)$ & $2.4(3)$ \\
\hline \hline
\end{tabular}




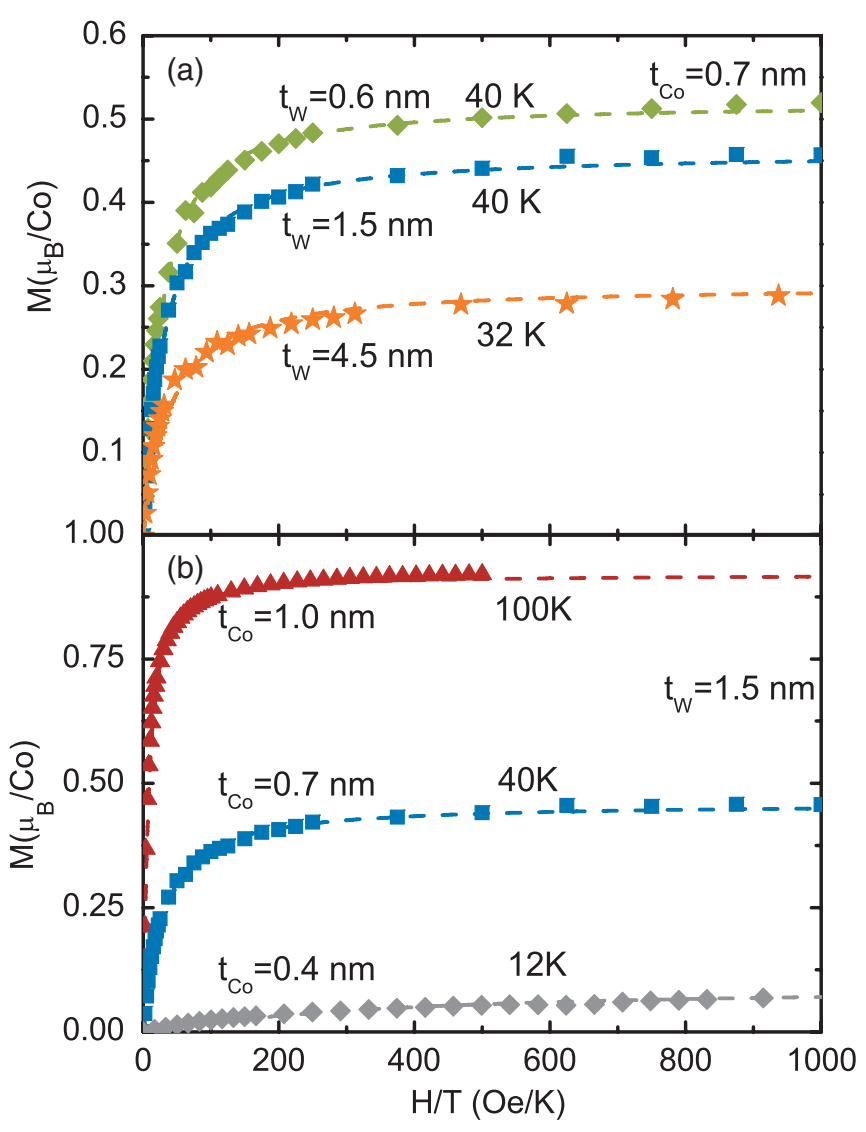

FIG. 12. (Color online) Magnetization curves for samples from the $a$ series in (a) and from the $b$ series in (b). The dashed lines are fitted Langevin curves with $\left\langle D_{\mathrm{mag}}\right\rangle$ and $\sigma$ parameters given in Table III.

An average $\mathrm{Co}_{1-x} \mathrm{~W}_{x}$ alloy composition in the Co-W NPs may be proposed by comparing the $m_{\text {at }}$ values to those measured for Co-W films deposited by rf sputtering in Ref. 34. Values of percent of $\mathrm{W}$ in the alloy are shown in Table III. The $\mathrm{W}$ concentration that explains the reduction in magnetic moment ranges from 18.2 to 20.3 at.\% in the $a$ samples series, and from 14.1 to 22.3 at.\% in the $b$ samples series.

From the equilibrium magnetic properties of each sample, we can determine the particle-size distribution and compare it with the results obtained by HRTEM and STEM. This is obtained by fitting the $M(H)$ curves at high $T$ to a Langevin function, averaged over the appropriate particle-size distribution. We define $g\left(D_{\mathrm{mag}}\right)$ as the Gaussian distribution of a number of particles having a diameter equal to $D_{\mathrm{mag}}$. For spherical particles, the particle magnetization is written as $m_{\mathrm{part}}=\pi M_{\mathrm{sb}} D_{\mathrm{mag}}^{3} / 6$, where $M_{\mathrm{sb}}$ is defined as the particle magnetic density, calculated from the saturation magnetization values and the density of the Co-W alloy formed on each sample as

$$
M_{\mathrm{sb}}(x)=\frac{m_{\mathrm{at}}\left(\mu_{B} / \mathrm{Co}\right) \rho(x)}{M_{\mathrm{w}}(x)} N_{a} \mu_{B},
$$

where $x$ gives the $\mathrm{Co}_{1-x} \mathrm{~W}_{x}$ alloy composition, $\rho(x)$ is the density and $M_{\mathrm{w}}(x)$ the molecular weight of the alloy, $N_{a}$ is the Avogadro number, and $\mu_{B}$ the Bohr magneton. Since there
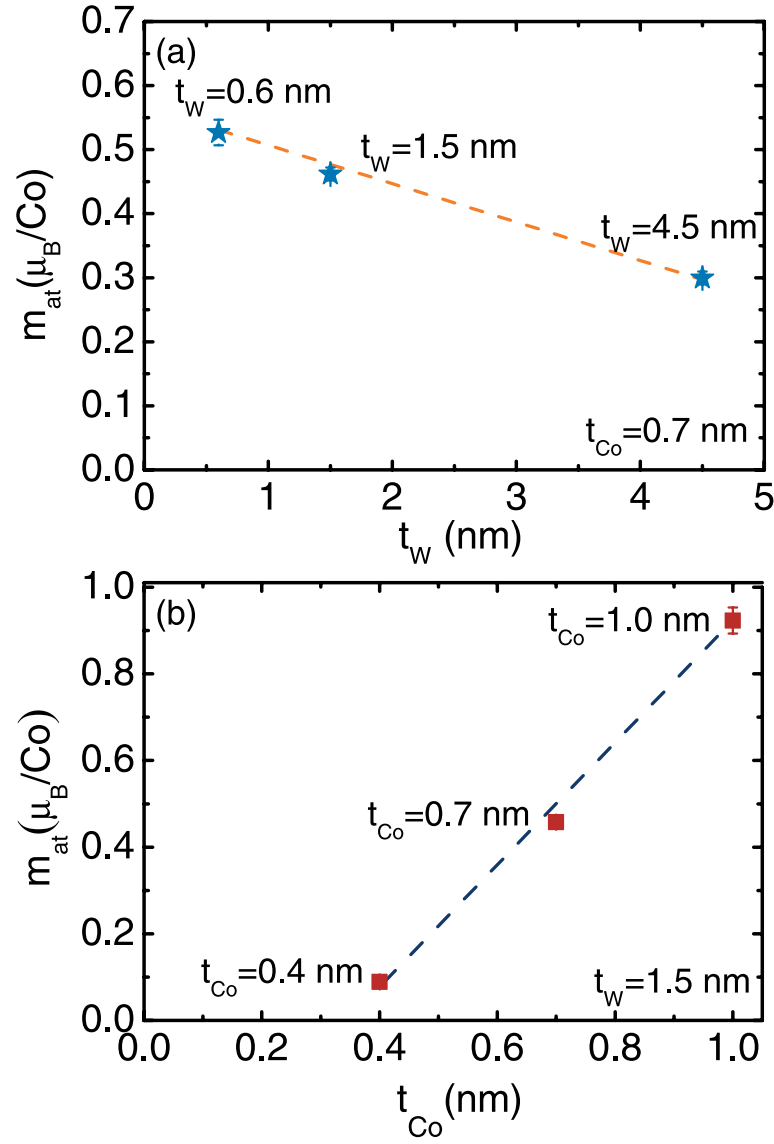

FIG. 13. (Color online) Total Co magnetic moment per Co atom in the Co-W NPs as deduced from the $M(H)$ measurements. The dashed lines are guides to the eye. (a) $a$ samples series. (b) $b$ samples series.

are no direct values in the literature for the densities of the Co-W alloys found for our samples, we need to calculate them. Knowing that the density for metallic Co is $8.9 \mathrm{~g} / \mathrm{cm}^{3}$ and the one for $\mathrm{Co}_{3} \mathrm{~W}$ alloy is $12.7 \mathrm{~g} / \mathrm{cm}^{3},{ }^{59}$ we can apply linear approximation for $\rho(x)$ versus $x$. Given the $x$ values found for our Co-W samples (listed in Table III), we can interpolate each composition value and get its respective density.

The experimental data are fitted by the expression

$$
\begin{aligned}
M(H, T)= & x_{\text {para }} m_{\text {para }}+\left(1-x_{\text {para }}\right) M_{s b} \\
& \times \frac{\int g\left(D_{\mathrm{mag}}\right) V\left[M(H, T, \mu) / m_{\mathrm{at}}\right] d D}{\int g\left(D_{\mathrm{mag}}\right) V d D_{\mathrm{mag}}} .
\end{aligned}
$$

Let us recall that $x_{\text {para }}$ gives the fraction of nonaggregated Co atoms present in the samples. In the case of bare and noblemetal capped Co NPs, they give a paramagnetic contribution as a positive slope of $M(H)$ at high fields. ${ }^{10,11}$ In our Co-W alloy NPs, the nonaggregated Co atoms are also alloyed with W. They do not contribute to the magnetic signal, i.e., at high fields, the slope of $M(H)$ is negligible. Then, $m_{\text {para }}$ in Eq. (2) is null, and Eq. (2) is reduced to the second term.

Results for these fits are the dashed lines in Fig. 12. The average magnetic diameter values $\left\langle D_{\mathrm{mag}}\right\rangle$ and the distribution width for the Gaussian distribution of particle size $\sigma_{\mathrm{mag}}$ obtained are listed in Table III. These parameters were confirmed by fitting the equilibrium susceptibility $1 / \chi_{\mathrm{eq}}(\mathrm{T})$ 
in the superparamagnetic temperature regime, measured for the same samples. That is, for a system of particles of that diameter and size distribution, $\chi_{\text {eq }}$ can be expressed as

$$
\chi_{\mathrm{eq}}=\frac{\int g\left(D_{\mathrm{mag}}\right) V\left(M_{\mathrm{sb}}^{2} V / 3 k_{B} T\right) d D_{\mathrm{mag}}}{\int g\left(D_{\mathrm{mag}}\right) V d D_{\mathrm{mag}}} .
$$

Comparing the values of $\left\langle D_{\text {eff }}\right\rangle$ obtained from the morphological analysis described in Sec. IV with the $\left\langle D_{\mathrm{mag}}\right\rangle$ derived from this magnetic analysis, we note that the latter is smaller. From the above facts, we may conclude that our amorphous Co-W NPs are composed by a magnetic Co-W alloy core surrounded by unalloyed metallic $\mathrm{W}$, which tends to fill the interparticle space, as described in Secs. IV and V.

\section{B. Magnetic anisotropy of the $\mathrm{Co}-\mathrm{W}$ particles}

The phenomenon of superparamagnetic blocking described in the FC-ZFC curves' analysis is related to the magnetic anisotropy of the particles. The anisotropy favors some particular orientations of the magnetic moment, two opposite to each other in the simplest case of uniaxial anisotropy, which are separated by activation energy barriers $U$. The rate at which the magnetic moment of the particles flips from one orientation to the other is determined by this anisotropy $K_{\text {eff }}$, so that we write $U=K_{\text {eff }} V$, with $V$ being the particle magnetic volume. The relaxation time $\tau$ follows an Arrhenius law

$$
\tau=\tau_{0} \exp \left(U / k_{B} T\right),
$$

where $\tau_{0} \approx 10^{-10}-10^{-13} \mathrm{~s}$ is the inverse attempt frequency that depends on the damping of the magnetic moments by the phonons. The superparamagnetic blocking occurs when $\tau=t_{e}$, where $t_{e}$ is the measuring time of each experimental point, so $T_{B} \cong \alpha U / k_{B} \ln \left(t_{e} / \tau_{0}\right)$, where $\alpha$ is a constant that depends on the width of the particle-size distribution.

An accurate way to obtain $U$ is derived from the full distribution of activation energies $f(U)$ and then to find which value of $U$ corresponds to particles with a diameter

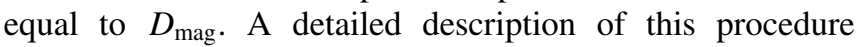
can be found in Ref. 10. $f(U)$ can be directly determined from the analysis of the imaginary part of $\chi_{\mathrm{ac}}(T)$, measured near the superparamagnetic blocking temperature $T_{B}$. For noninteracting particles, we write

$$
\chi^{\prime \prime} \simeq \frac{\pi}{2} k_{B} T \chi_{\mathrm{eq}}(T, U) f\left(U_{b}\right),
$$

where we define $U_{b}=k_{B} T \ln \left(1 / \omega \tau_{0}\right)$ as a scaling variable, corresponding to the activation energy of those particles having $\tau=t_{e}=1 / \omega$ at a given $T$. The frequency dependent $\chi_{\mathrm{ac}}$ should converge into a single curve when plotting $\chi^{\prime \prime}$ versus $U_{b} / k_{B} \cdot f\left(U_{b}\right)$ can be determined from Eq. (5) from a $\chi^{\prime \prime}\left(U_{b}\right)$ fit. In Fig. 14, we have plotted the result obtained from this $\chi_{\mathrm{ac}}(T)$ analysis, including the $\chi^{\prime \prime}\left(U_{b}\right)$ fit, for the sample with $t_{\mathrm{Co}}=0.7 \mathrm{~nm}$ and $t_{\mathrm{W}}=1.5 \mathrm{~nm}$. We also show the Arrhenius plot of $\ln (1 / \omega)$ versus $1 / T_{B}$ from the analysis obtained from $\chi^{\prime \prime}$ measurements for the $a$ series samples. In the case of the sample with the lowest amount of Co $\left(t_{\mathrm{Co}}=0.4 \mathrm{~nm}\right.$ and $t_{\mathrm{W}}=$ $1.5 \mathrm{~nm})$, this analysis was not performed since the $\chi_{\mathrm{ac}}$ signal was negligible.

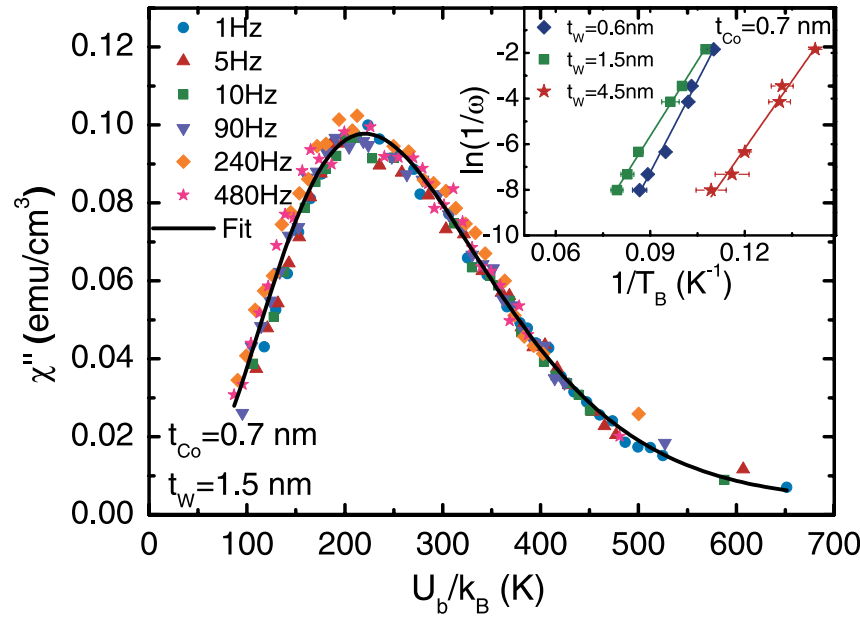

FIG. 14. (Color online) Imaginary part of the ac susceptibility for the sample with $t_{\mathrm{Co}}=0.7 \mathrm{~nm}$ and $t_{\mathrm{W}}=1.5 \mathrm{~nm}$ plotted as a function of the scaling variable $U_{b}$. The solid line is the fitting result. Inset: Arrhenius plot of the measuring time $(1 / \omega)$ as a function of $T_{B}$, as obtained from $\chi^{\prime \prime}$ measurements for the $a$ series samples.

$K_{\text {eff }}$ is then obtained from $U\left(\left\langle D_{\text {mag }}\right\rangle\right)=K_{\text {eff }}(\pi / 6)\left\langle D_{\text {mag }}\right\rangle^{3}$, where $\left\langle D_{\text {mag }}\right\rangle$ denotes the average magnetic diameter of the spherical particles. All this analysis is performed assuming that the magnetic moment is just supported by the $\mathrm{Co}-\mathrm{W}$ magnetic alloy core. The values of the average $\langle U\rangle$ and $K_{\text {eff }}$ obtained for all samples are listed in Table III.

$K_{\text {eff }}$ in our Co-W NPs decreases with increasing $t_{\mathrm{W}}$ for the $a$ series samples and with decreasing $t_{\text {Co }}$ for the $b$ series samples. This gives $K_{\text {eff }}$ a trend directly proportional to $\left\langle D_{\mathrm{mag}}\right\rangle$, suggesting that surface anisotropy is not playing an important role in the $K_{\text {eff }}$ as in other particulate systems. ${ }^{4,11,14,25-27}$ Further discussion on these results will be presented in Sec. VIII.

\section{MAGNETIC STUDY: X-RAY MAGNETIC CIRCULAR DICHROISM MAGNETOMETRY}

$\mathrm{XMCD}$ is a very powerful technique that allows us to perform an element selective magnetometry on our $\mathrm{Co}-\mathrm{W}$ NPs. From an XMCD experiment at the $L_{2,3}$ edges of Co, one obtains, through the sum rules, ${ }^{60,61}$ the spin and orbital moments averaged over the volume of the particle, and on the number of holes $n_{h}$ in the empty exchange split $n d$ subbands (Co $3 d$ in the present case). The sum rules have been applied to the spectra to derive the orbital, spin, and total magnetic moments per $3 d$ hole of Co, $m_{\mathrm{L}} / n_{h}, m_{\mathrm{S}} / n_{h}$, and $m_{\mathrm{at}} / n_{h}$, respectively, with the symbols and sign criteria defined in Ref. 62. We define the constant $C=\left(I_{L 2}+I_{L 3}\right)$, where $I_{L 2}$ and $I_{L 3}$ are the white-line-integrated intensities after subtraction of the contributions from transitions to the continuum. It is noteworthy that, in the present case of amorphous particles, with their individual anisotropy axes oriented at random, the dipolar term $m_{D}$ cancels out so that the sum rule $(A-2 B)=$ $-C m_{\mathrm{S}} /\left(n_{h} \mu_{B}\right)$ yields the spin component $m_{\mathrm{S}} / n_{h} . A$ and $B$ are the integrated areas of the $L_{3}$ and $L_{2}$ XMCD peaks, respectively. Aside from this, from the ratio of the sum rules, $m_{\mathrm{L}} / m_{\mathrm{S}}$ is directly derived, which is independent of $n_{h}$ and of the normalization method used. 
$\mathrm{XMCD}$ also allows us to study the changes in the orbital magnetic moment in the Co-W NPs samples as a function of $t_{\mathrm{W}}$ and $t_{\mathrm{Co}}$ by obtaining the average $m_{\mathrm{L}} / n_{h}$ per Co atom present in the sample. In the experiments, a magnetic field of $20 \mathrm{kOe}$ is applied, sufficient to saturate the particle spin magnetization. The contribution of the orbital moment to the XMCD signal is the averaged projection of the local orbital magnetic moments in the direction of the applied field (parallel to the photon beam direction in the experiments).

The experimental XMCD spectra at the Co $L_{2,3}$ edges for sample with $t_{\mathrm{Co}}=0.7 \mathrm{~nm}$ and $t_{\mathrm{W}}=1.5 \mathrm{~nm}$ is depicted in Fig. 15 , together with its XAS, for normalization sake. All XMCD results are qualitatively similar, although the actual derived $A$ and $B$ values differ. The derived $m_{\mathrm{L}} / m_{\mathrm{S}}, m_{\mathrm{L}} / n_{h}$, and $m_{\mathrm{S}} / n_{h}$ values are given in Table IV, along with the values of the total moment $m_{\text {at }} / n_{h}$.

Self-saturation effects may affect these TEY measurements in principle. It is customarily corrected in thin films, ${ }^{63}$ and has been done in the case of rather large $\mathrm{Co}$ and $\mathrm{Fe}$ nanoparticles. ${ }^{64,65}$ To correct for this effect, the electron escape depth parameter $\lambda_{e}$ at low kinetic energies of the particular material is needed. The Co-W alloy's $\lambda_{e}$ at low energies is not known, but at higher energies, of the order of $100 \mathrm{eV}$, it is very near to that for Co. ${ }^{66}$ Under this hypothesis, the correction due to saturation effects would be very similar to that needed for Co particles of the same size. It has been estimated that
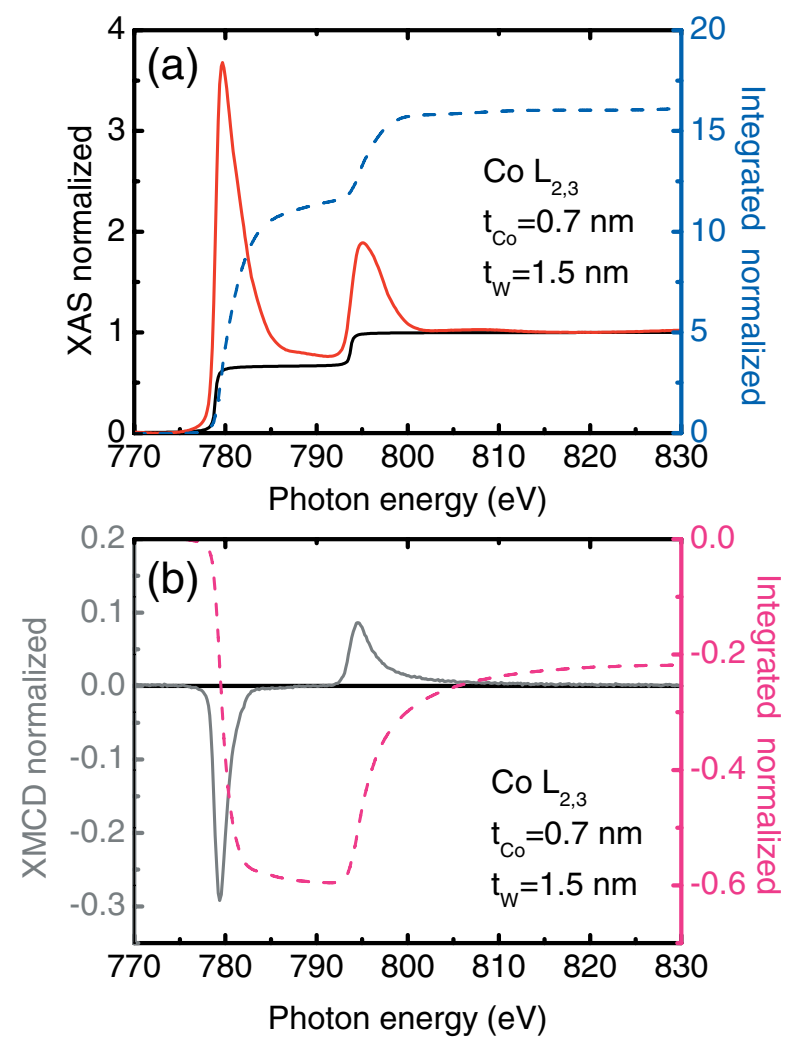

FIG. 15. (Color online) Co- $L_{2,3}$ edges spectra of the $t_{\mathrm{Co}}$ at $0.7 \mathrm{~nm}$, $t_{\mathrm{W}}=1.5 \mathrm{~nm}$ sample measured at $T=5 \mathrm{~K}, \mu_{0} H=2 \mathrm{~T}$, and incident angle $10^{\circ}$. (a) Normalized XAS spectra mean curve (solid line). The two-step function used is also indicated. Integrated white line (dashed line). (b) Normalized XMCD spectra (solid line), integrated area (dashed line).
TABLE IV. $m_{\text {at }} / n_{h}, m_{\mathrm{L}} / n_{h}, m_{\mathrm{S}} / n_{h}$, and $m_{\mathrm{L}} / m_{\mathrm{S}}$ per Co atom as determined from XMCD measurements. For the fcc bulk Co, $m_{\mathrm{L}} / m_{\mathrm{S}}=0.078$ (Ref. 67), $m_{\mathrm{L}} / n_{h}=0.134$, and $m_{\mathrm{S}} / n_{h}=0.248 \mu_{B}$ per $3 d$ hole (Ref. 68) $\left(3 d n_{h}^{\text {Co }}=2.49\right)$ (Ref. 69).

\begin{tabular}{lcccccc}
\hline \hline $\begin{array}{l}\text { Samples } \\
\text { series }\end{array}$ & $\begin{array}{c}t_{\mathrm{Co}} \\
(\mathrm{nm})\end{array}$ & $\begin{array}{c}t_{\mathrm{W}} \\
(\mathrm{nm})\end{array}$ & $m_{\mathrm{L}} / m_{\mathrm{S}}$ & $\begin{array}{c}m_{\mathrm{L}} / n_{h} \\
\left(\mu_{B}\right)\end{array}$ & $\begin{array}{c}m_{\mathrm{S}} / n_{h} \\
\left(\mu_{B}\right)\end{array}$ & $\begin{array}{c}m_{\mathrm{at}} / n_{h} \\
\left(\mu_{B}\right)\end{array}$ \\
\hline$a$ & 0.7 & 0.6 & $0.082(1)$ & $0.018(1)$ & $0.216(3)$ & $0.234(3)$ \\
& 0.7 & 1.5 & $0.085(1)$ & $0.015(1)$ & $0.172(4)$ & $0.186(4)$ \\
& 0.7 & 4.5 & $0.079(2)$ & $0.010(1)$ & $0.120(5)$ & $0.130(6)$ \\
$b$ & 0.4 & 1.5 & $0.159(7)$ & $0.007(1)$ & $0.042(4)$ & $0.048(4)$ \\
& 0.7 & 1.5 & $0.085(1)$ & $0.015(1)$ & $0.172(4)$ & $0.186(4)$ \\
& 1.0 & 1.5 & $0.071(1)$ & $0.021(1)$ & $0.290(9)$ & $0.310(9)$ \\
\hline \hline
\end{tabular}

for Co particles with diameter $D=3 \mathrm{~nm}$, the $m_{\mathrm{S}}$ and $m_{\mathrm{L}}$ parameters obtained with the sum rules from uncorrected XAS and XMCD spectra may be underestimated by $2 \%$ and $13 \%$, respectively ${ }^{64}$ which amounts to an underestimation of $3 \%$ in $m_{\text {at }}$. Such a difference is comparable to the other sources of error in these experiments, such as the presence of $x_{\text {para }}$ or the size distribution of the particles. Therefore, to account for this effect, we have added this source of error to the total estimated errors in the Table IV data, and should take conclusions from the trends rather than from the absolute values.

The variation of the $m_{\mathrm{L}} / m_{\mathrm{S}}$ ratio as a function of the amount of $\mathrm{Co}$ and $\mathrm{W}$ in the samples can be analyzed from the values listed in Table IV. In $b$ samples series, for $t_{\mathrm{Co}}=0.4 \mathrm{~nm}$, the ratio is much larger than the value for bulk Co $\left(m_{\mathrm{L}} / m_{\mathrm{S}}=\right.$ $0.078)$, and decreases toward that value as the $t_{\mathrm{Co}} / t_{\mathrm{W}}$ ratio increases, being quite close for $t_{\mathrm{Co}}=1.0 \mathrm{~nm}\left(m_{\mathrm{L}} / m_{\mathrm{S}}=\right.$ 0.071) (see Table IV). The reason for this difference at low $t_{\mathrm{Co}} / t_{\mathrm{W}}$ can be found in the unusually low value of $m_{\mathrm{S}} / n_{h}$ $\left(0.042 \mu_{B}\right.$ per $3 d$ hole) for $t_{\mathrm{Co}}=0.4 \mathrm{~nm}$. Although the $m_{\mathrm{L}} / n_{h}$ decreases with $\mathrm{W}$ alloying, the reduction of $m_{\mathrm{S}} / n_{h}$ is much stronger [see Fig. 16(b)]. Therefore, alloying with W affects essentially the difference between up- and down-filled states in the Co $3 d$ band. In contrast, the $m_{\mathrm{L}} / m_{\mathrm{S}}$ ratio as a function of the W-capping thickness is practically constant [see Table IV, and parallel variation of $m_{\mathrm{L}} / n_{h}$ and $m_{\mathrm{S}} / n_{h}$ in Fig. 16(a)], i.e., the compositional $\mathrm{Co} / \mathrm{W}$ ratio in the alloys obtained in the $a$ samples series is similar (see also alloys composition in Table III). It may be concluded that the crucial factor to reach the different alloys in the Co-W NPs depends more on the amount of Co available for alloying than on the amount of $\mathrm{W}$ in the sample.

We may estimate $n_{h}$ for each Co-W composition by substituting in the $m_{\mathrm{at}} / n_{h}$ values given in Table IV those $m_{\text {at }}$ determined from $M(H)$ measurements in the previous section (see Table III). Although W may be polarized by Co, its contribution to the net magnetization is orders of magnitude lower, ${ }^{57,70}$ so it is considered as negligible in this analysis. Therefore, the magnetization of our samples is mainly due to the Co present in the $\mathrm{Co}-\mathrm{W}$ particles. We may assume that both SQUID and XMCD magnetometry techniques yield the average total Co moment at saturation (for the sake of comparison, we have used values at $H=20 \mathrm{kOe}$ ). Thus, from the comparison of both values, we can extract the variation of the $n_{h}$ in the Co $3 d$ band in the Co-W NPs. For the $a$ series 

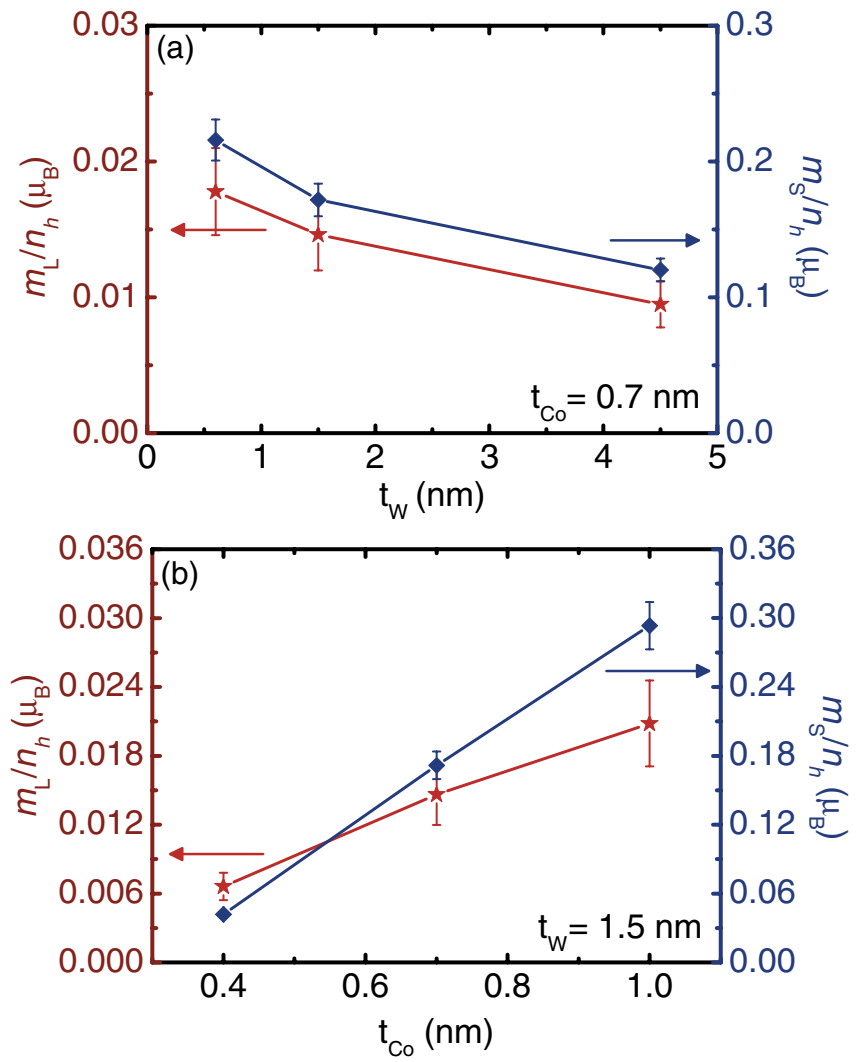

FIG. 16. (Color online) Dependence of Co $m_{\mathrm{L}} / n_{h}$ and $m_{\mathrm{S}} / n_{h}$ on the $\mathrm{Co}$ and $\mathrm{W}$ composition. (a) $a$ samples series. (b) $b$ samples series.

samples, $n_{h}$ was found to be around an almost constant value of $n_{h}=2.4(1)$. Within experimental errors, this result is on the order or slightly lower than that for bulk $\operatorname{Co}\left(n_{h}=2.49\right),{ }^{69}$ and is well in agreement with the insignificant variation observed in the pre-edge peak of Co $K$ XAS spectra for the same samples, described in Sec. V, i.e., the Co $3 d-\mathrm{W} 5 d$ hybridization is hardly affected by small variations of the amount of $\mathrm{W}$ for a fixed $\mathrm{Co}$, showing once again that the alloy composition for the three samples along this series is similar. For the $b$ series samples, $n_{h}$ was found to vary within $20 \%$ of the average value found for the $a$ series. The general trend was that of increase as the amount of Co in the sample increases for a fixed $t_{\mathrm{W}}$ value. This latter result is also in agreement to what was observed at the Co $K$-edge XAS measurements in this samples series since it gives evidence of increasing localization of the $3 d$ states in $\mathrm{Co}$ as the $\mathrm{Co} / \mathrm{W}$ ratio in the sample increases.

\section{DISCUSSION}

We have found that the magnetic moment per Co atom in our alloy decreases with increasing W content. Such dependence has been extensively reported both in crystalline and amorphous alloys following the formula $T_{1-x} M_{x}$, with $T$ usually being $\mathrm{Fe}, \mathrm{Co}$, or $\mathrm{Ni}$, and $M$ an early transition metal that favors the amorphicity in the alloy. ${ }^{58} \mathrm{~A}$ generalized Slater-Pauling model based on the existence of a gap, or minimum, in the conduction-band density of states has been used to describe this trend. ${ }^{58}$ Such a gap tends to conserve the number of conduction electrons in an alloy series leading to a

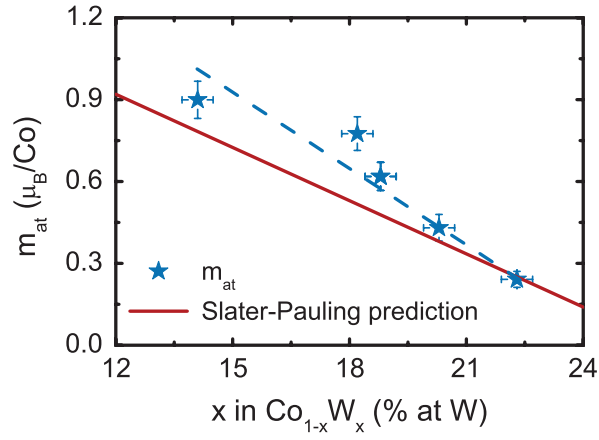

FIG. 17. (Color online) Dependence of $m_{\text {at }}$ with the composition of the Co-W alloy in the NPs. The dashed line is a guide to the eye.

linear variation of $m_{\text {at }}$ (the atom-averaged magnetic moment of the alloy) with $Z_{M}$ (the atomic valence of the $M$ metal $Z_{M}=6$ for W). ${ }^{58}$ The relation of $m_{\text {at }}$ as a function of $x$ is then expressed as

$$
m_{\mathrm{at}}(x)=m_{\text {metal }}-x\left(10+Z_{M}-Z_{T}\right) \mu_{\mathrm{B}},
$$

where $Z_{T}$ and $m_{\text {metal }}$ represent the atomic valence and magnetic moment in the pure $T$ metal $\left(Z_{T}=9\right.$ and $m_{\text {metal }}=$ $1.7 \mu_{B}$ for Co). Strong reduction of $m_{\mathrm{at}}$ is expected when $M$ is a nonmagnetic element. Comparison of this theoretical prediction with the values obtained for our Co-W NPs is shown in Fig. 17. Although there is a qualitative agreement in the trends, we find a significant deviation from the predicted slope, which can be attributed to the limitations of the model that takes insufficient account of the atomic structure, nearestneighbor environments, and chemical disorder. ${ }^{58}$

In $\mathrm{Co}$ amorphous magnetic alloys, which have local anisotropy at the Co sites with randomly oriented local easy axes, the Co-Co exchange interaction is of sufficient intensity to guarantee collective ferromagnetic order if the Co content is above a critical value. ${ }^{58}$ In $\mathrm{Co}-\mathrm{W}$ alloys, it is $\sim 76$ at.\% Co, so that those alloys with smaller values have no magnetic moment. ${ }^{34}$ In general, for $3 d$-metal-based alloys, the orientational correlation length of the local magnetic moments has been estimated to be of the order of a few $\mu \mathrm{m} .{ }^{71}$ Therefore, in $\mathrm{Co}-\mathrm{W}$ nanoparticles, with a diameter of the order of a few nanometers, exchange stiffness can maintain local moment correlation within the whole particle, and, thus, yield to a particle net average moment and anisotropy.

Amorphous magnetic alloys are expected to behave magnetically isotropic or to exhibit a low anisotropy (in the case of thin films where a stress-induced anisotropy can be present) given their lack of atomic long-range order. In contrast, in our Co-W NPs, $K_{\text {eff }}$ is all but negligible. Its origin may be traced back to the structural or chemical SRO we have shown to exist in Sec. V. Indeed, some amorphous alloys, typically following the formula $T_{1-x} M_{x}$, still exhibit remarkable SRO. ${ }^{41,55}$ Coordination numbers of about 12 found by structural characterization of these alloys may be visualized as hep or fcc like. ${ }^{55}$ Such amorphous materials are often simulated by small structural units, which are defined by a reference atom and its nearest neighbors. The structural model should contain sites having hcp- and fcc-like symmetries and are modeled as icosahedral, octahedral, and trigonal units. $^{41}$ Given the presence of SRO, large local 
magnetic anisotropies, comparable to those of crystalline Co, for example, are expected. Moreover, if the anisotropy is due to chemical SRO, $K_{\text {eff }}$ is expected to increase linearly with the $\mathrm{W}$ concentration, ${ }^{38}$ contrary to our observation. The origin of $K_{\text {eff }}$ in these materials is then structural. Values of $K_{\text {eff }}$ on the order of the $10^{5} \mathrm{erg} / \mathrm{cm}^{3}$ have been reported for amorphous $\mathrm{Co}_{1-x} \mathrm{Ti}_{x}$ films ${ }^{37}$ and on the order of $10^{6} \mathrm{erg} / \mathrm{cm}^{3}$ for amorphous $\mathrm{Co}_{1-x} \mathrm{Zr}_{x}$ films. ${ }^{38}$ The latter is comparable to those obtained for our amorphous Co-W alloy NPs. Such high values of magnetic anisotropy are attributed to the strong local uniaxial anisotropy of the clusters. ${ }^{37,38} \mathrm{In} \mathrm{Co}_{1-x} M_{x}$ amorphous alloys $(M=\mathrm{Ti}$ and $\mathrm{Zr}$ ), clusters formed of only Co atoms are magnetically isotropic, while those where the Co atom possess a number of $M$ atoms as nearest neighbors present a local anisotropy, so submitted to greater anisotropy energy. The local anisotropy depends on both the local symmetry of the Co atoms and to the saturation magnetization $M_{\mathrm{S}} \cdot{ }^{38}$ If the local disorder increases by increasing the amount of $M$ atoms, the clusters are less defined, giving rise to a reduction in $K_{\text {eff }}$. Such dependence of $K_{\text {eff }}$ with $x$ has been observed in $\mathrm{Co}_{1-x} \mathrm{Ti}_{x}$ (Ref. 37) and $\mathrm{Co}_{1-x} \mathrm{Zr}_{x}$ films, ${ }^{38}$ and is also observed in our Co-W NPs (see Fig. 18).

In Fig. 19, the $K_{\text {eff }}$ and $m_{\mathrm{L}} / m_{\mathrm{S}}$ results found for the amorphous Co-W NPs are depicted together with the values found for bulk Co, and NPs of the same average diameter ( $D \sim 3 \mathrm{~nm}$ ); the bare Co NPs (Ref. 14) and, for completeness, the published values for alloyed Co-Pt particles, are included. ${ }^{16}$ In all these cases, hybridization effects between $3 d$ Co and $5 d$ metal bands are at the origin of the increase of anisotropy. Thus, alloying Co with $\mathrm{W}$ increases the anisotropy with respect to bulk Co where crystalline anisotropy is dominant. The larger $K_{\text {eff }}$ values found in the Co-Pt alloyed particles is due to the larger spin-orbit coupling constant in the Pt case with respect to the $\mathrm{W}$ one $\left[\xi_{5 d}=0.66\right.$ and $0.038 \mathrm{eV}$ for Pt (Ref. 72) and $\mathrm{W}$ (Ref. 73), respectively]. However, the enhancement driven by surface effects observed in the bare pure Co NPs is significantly larger. ${ }^{14}$

Aside from this, when plotting $K_{\text {eff }}$ versus the diameter of the Co-W particles (Fig. 20), we find an increase of $K_{\text {eff }}$ with increasing the NP diameter instead of the $1 / D$ dependence found for the bare Co NPs. ${ }^{11}$ This feature implies that there is an increase in the intrinsic anisotropy of the alloy, while the surface anisotropy is zero, as occurs, for example, in Co-Pt alloy NPs. ${ }^{16}$

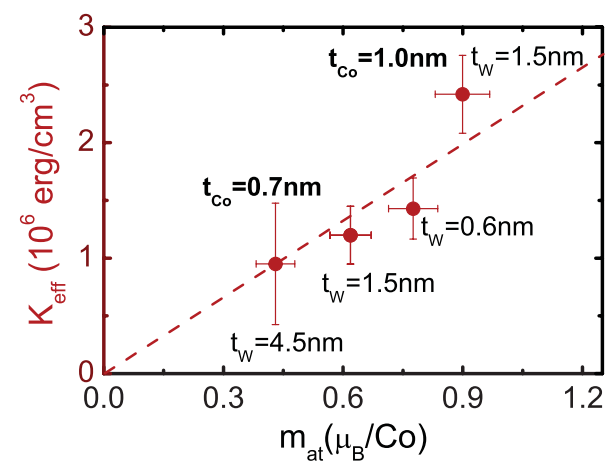

FIG. 18. (Color online) Dependence of $K_{\text {eff }}$ with $m_{\text {at }}$ for all Co-W NPs samples. The dashed line is a guide to the eye.

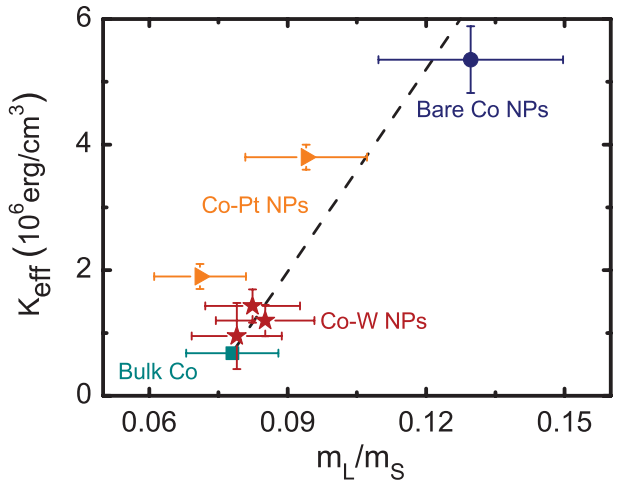

FIG. 19. (Color online) Dependence of $K_{\text {eff }}$ with $m_{\mathrm{L}} / m_{\mathrm{S}}$ for the $a$ series of Co-W samples and comparison with those for the bulk Co (Ref. 67), bare Co NPs with $t_{\mathrm{Co}}=0.7 \mathrm{~nm}$ (Ref. 14), and Co-Pt NPs with comparable diameters (Ref. 16). The dashed line is a guide to the eye.

When comparing the magnetic behavior of our amorphous Co-W NPs with some other amorphous NP systems studied in the literature, we find a quite different magnetic behavior. Most of the amorphous Fe NPs, ${ }^{18,19,21} \mathrm{Fe}$ oxides, ${ }^{20,22}$ organic coated Fe NPs, ${ }^{23,24}$ amorphous Ni powder, ${ }^{28} \mathrm{Fe}-\mathrm{Ni}$ alloys, ${ }^{29}$ and $\mathrm{NiFe}_{2} \mathrm{O}_{4}$ (Ref. 30) are prepared by sonochemical methods, which lead to agglomeration of the particles. Thus, in such systems, the magnetic interactions between particles play an important role. Instead, our $\mathrm{Co}-\mathrm{W}$ NPs system constitute an ideal example of uniform noninteracting amorphous NPs well dispersed in an insulating matrix. Properties of the amorphous $\mathrm{Fe}$ - and Ni-based NP systems are also those of superparamagnetic particles, with blocking temperature depending on the particle size. ${ }^{18-24,28-30}$ In the organic-coated Fe NPs, the surface dominates their magnetic properties since there is a chemical bonding between the functional groups attached to the particle and the Fe atoms. ${ }^{23,24}$ The chemical bonding modifies their $T_{\mathrm{B}}$ and saturation magnetization $M_{\mathrm{S}}$. However, in the case of our Co-W NPs, we have demonstrated that surface contribution is not as important as in those particulate systems.

Amorphous boride NPs with formula TM-B (with TM being one or two transition metals) show superparamagnetic behavior, interpreted in the frame of a core-shell model. ${ }^{4,25-27}$

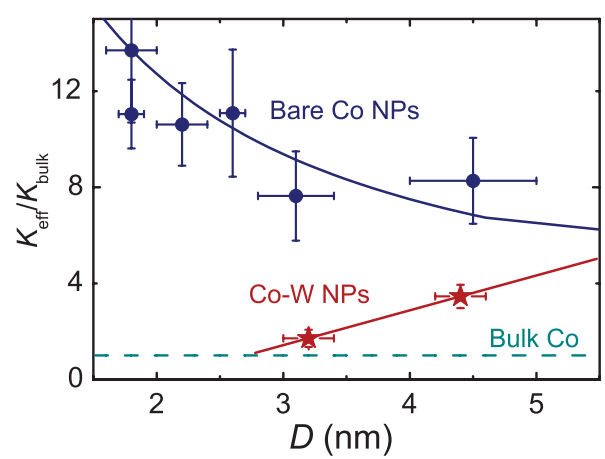

FIG. 20. (Color online) Average magnetic anisotropy normalized to the Co bulk value $\left(K_{\text {bulk }}=7 \times 10^{5} \mathrm{erg} / \mathrm{cm}^{3}\right)$ (Ref. 74$)$ as a function of the Co-W particle diameter for the $b$ samples series along with the values for bare Co NPs (Ref. 11). 
Such particles are prepared by chemical synthesis and dispersed in a polymeric matrix for measurements. Opposite to our $\mathrm{Co}-\mathrm{W}$ NPs, TM-B have a distinct high-temperature blocking temperature $T_{\mathrm{B}}$, assigned to blocking of the ferromagnetically ordered core spins, and a second one at lower temperature, explained as due to blocking of the disordered shell spins. Besides, the $M(H)$ curves were temperature dependent, i.e., they did not follow a corresponding state law with $H / T$, on one hand, and had two components, a typical Langevin dependence, contributed by the core, and a lineardependent contribution, contributed by the spin disordered shell, on the other. In contrast, our Co-W NPs do fulfill the corresponding state law and such a core-shell model (each with a different magnetic contribution) is not applicable.

In other $\mathrm{Co}-\mathrm{W}$ multilayer systems, the origin of the magnetic anisotropy observed is ascribed to the surface of the Co layers ${ }^{75,76}$ and to volume-type strain anisotropy. ${ }^{75}$ However, we have demonstrated that interface effects play no role in the increase of $K_{\text {eff }}$ obtained for our Co-W NPs, compared to the bulk Co value.

Our Co-W NPs have an oblate shape with semiaxes $a=\left\langle D_{\mathrm{eff}}\right\rangle_{\mathrm{PV}} / 2$ and $c=\left\langle D_{\mathrm{eff}}\right\rangle_{\mathrm{CS}} / 2$, albeit with a small $c / a$ ratio; in the two samples for which the plan-view and crosssection average diameter could be determined (see Table I), $c / a \approx 0.93(1)$. Then, one may estimate the shape anisotropy constant $^{77}$ as

$$
K_{\mathrm{sh}}=\frac{1}{2}\left(N_{a}-N_{c}\right) M_{\mathrm{sb}}^{2}
$$

where the demagnetization factors for that $c / a$ ratio are $N_{a} \approx 0.325$ and $N_{c} \approx 0.350,{ }^{78}$ for the long- and short-particle semiaxes, and $M_{\mathrm{sb}}$ is the magnetization density of the particle. For the two particles with $t_{\mathrm{Co}}=0.7 \mathrm{~nm}$ and $t_{\mathrm{W}}=0.6$ and $1.5 \mathrm{~nm}, M_{\mathrm{sb}}=0.413$ and $0.367 \times 10^{3} \mathrm{emu} / \mathrm{cm}^{3}$, respectively (see Table III), which yields $K_{\mathrm{sh}}=-2.0$ and $-1.7 \times 10^{3}$ $\mathrm{emu} / \mathrm{cm}^{3}$, respectively. These values are nearly three orders of magnitude smaller than the anisotropy constant found from our ac susceptibility measurements, so, we may consider this contribution as negligible. Then, shape anisotropy can not explain the $K_{\text {eff }}$ found for our Co-W NPs. Stress anisotropy is also one order of magnitude smaller. ${ }^{58}$ Instead, the SRO detected in our amorphous Co-W alloy NPs is probably the main factor in the origin of their magnetic anisotropy.

Concluding, we have presented a complete study on a system of self-organized amorphous Co-W alloy nanoparticles, uniformly dispersed in an alumina matrix. The size and composition of the Co-W alloy NPs have been controlled by varying the amount of $\mathrm{Co}$ or $\mathrm{W}$ in the system. Such parameters strongly affect their structural and magnetic properties. Co-W hybridization and electronic transfer from W $5 d$ to Co orbitals have been observed by XANES measurements, so that as the $\mathrm{Co} / \mathrm{W}$ ratio in the samples increases, there is more electronic transfer from W $5 d$ toward Co $4 p$ bands and less delocalization of Co $3 d$ empty states. The amorphous nature of the Co-W NPs was verified by HRTEM and EXAFS measurements. The particles are superparamagnetic with minimal interactions between them, as demonstrated by the FC-ZFC magnetic measurements. Their magnetism is governed by the Co present in the alloy particles, so we were able to obtain values of the Co magnetic moment in the system via conventional SQUID magnetometry and compare it to results from XMCD measurements. A reduction of the Co moment per Co atom present in the samples was observed, compared to the bulk Co value. We have explained such a decrease as due to the reduction of Co coordination in the alloy, which was verified by EXAFS and has been described in the literature. SRO in the amorphous Co-W alloy NPs is evident and is responsible for the magnetic anisotropy detected in the particles, contrary to other amorphous nanoparticle systems where the anisotropy is due to surface effects. With this work, we supply a paradigmatic example in the phenomenological body of magnetic nanoparticle behavior.

\section{ACKNOWLEDGMENTS}

We thank G. Subías for her valuable discussions about the EXAFS measurements and analysis. The financial support of MICINN-FEDER MAT08/1077 and Aragonese IMANA project is acknowledged. A.I.F. acknowledges a CSIC JAE2008-Predoc grant. ESRF beamtime corresponds to HE1214, HE2238, HE2486, HE2541, and HE2952 experiments.

\footnotetext{
*figueroa@unizar.es

${ }^{1}$ M. Jamet, W. Wernsdorfer, C. Thirion, D. Mailly, V. Dupuis, P. Mélinon, and A. Pérez, Phys. Rev. Lett. 86, 4676 (2001).

${ }^{2}$ J. I. Martín, J. Nogués, K. Liuc, J. L. Vicente, and I. K. Schuller, J. Magn. Magn. Mater. 256, 449 (2003).

${ }^{3}$ J. Bansmann, S. H. Baker, C. Binns, J. A. Blackman, J. P. Bucher, J. D. Dávila, V. Dupuis, L. Favre, D. Kechrako, A. Kleibert, K. H. Meiwes-Broer, G. M. Pastor, A. Perez, O. Toulemonde, K. N. Trohidou, J. Tuaillon, and Y. Xie, Surf. Sci. Rep. 56, 189 (2005).

${ }^{4}$ J. Dormann, D. Fiorani, and E. Tronc, Adv. Chem. Phys. 98, 283 (1997).

${ }^{5}$ S. I. Woods, J. R. Kirtley, S. Sun, and R. H. Koch, Phys. Rev. Lett. 87, 137205 (2001).

${ }^{6}$ P. E. Jonsson, Adv. Chem. Phys. 128, 191 (2004).
}

${ }^{7}$ S. Bedanta and W. Kleemann, J. Phys. D: Appl. Phys. 42, 013001 (2009).

${ }^{8}$ F. Luis, F. Petroff, J. M. Torres, L. M. García, J. Bartolomé, J. Carrey, and A. Vaurès, Phys. Rev. Lett. 88, 217205 (2002).

${ }^{9}$ B. D. Terris and T. Thomson, J. Phys. D: Appl. Phys. 38, R199 (2005).

${ }^{10}$ F. Luis, J. M. Torres, L. M. García, J. Bartolomé, J. Stankiewicz, F. Petroff, F. Fettar, J. L. Maurice, and A. Vaures, Phys. Rev. B 65, 094409 (2002).

${ }^{11}$ F. Luis, F. Bartolomé, F. Petroff, J. Bartolomé, L. M. García, C. Deranlot, H. Jaffrès, M. J. Martínez, P. Bencok, F. Wilhelm, A. Rogalev, and N. Brookes, Europhys. Lett. 76, 142 (2006).

${ }^{12}$ J. Bartolomé, F. Luis, F. Petroff, F. Bartolomé, L. M. García, V. Cross, and H. Jaffres, Phys. Met. Metallogr. 99, S8 (2005), 
2nd Euro-Asian Symposium on Trends in Magnetism (EASTMAG2004), Krasnoyarsk State Univ, Krasnoyarsk, RUSSIA, AUG 24-27, 2004.

${ }^{13}$ J. Bartolomé, F. Bartolomé, L. M. García, F. Luis, F. Petroff, V. Cros, H. Jaffrès, and A. Vaurès, in Smart Materials for Ranging Systems, NATO Science Series, Vol. 226, edited by J. Franse, V. Eremenko, and V. Sirenko (Springer, The Netherlands, 2006), pp. 1-25.

${ }^{14}$ J. Bartolomé, L. M. García, F. Bartolomé, F. Luis, R. López-Ruiz, F. Petroff, C. Deranlot, F. Wilhelm, A. Rogalev, P. Bencok, N. B. Brookes, L. Ruiz, and J. M. González-Calbet, Phys. Rev. B 77, 184420 (2008).

${ }^{15}$ P. Bruno, Phys. Rev. B 39, 865 (1989).

${ }^{16}$ F. Tournus, A. Tamion, N. Blanc, A. Hannour, L. Bardotti, B. Prével, P. Ohresser, E. Bonet, T. Epicier, and V. Dupuis, Phys. Rev. B 77, 144411 (2008).

${ }^{17}$ K. H. J. Buschow, P. van Engen, and R. Jongebreur, J. Magn. Magn. Mater. 38, 1 (1983).

${ }^{18}$ M. W. Grinstaff, M. B. Salamon, and K. S. Suslick, Phys. Rev. B 48, 269 (1993).

${ }^{19}$ X. Cao, Y. Koltypin, G. Kataby, R. Prozorov, and A. Gedanken, J. Mater. Res. 10, 2952 (1995).

${ }^{20}$ X. Cao, Y. Koltypin, R. Prozorov, G. Katabya, and A. Gedanken, J. Mater. Chem. 7, 2447 (1997).

${ }^{21}$ K. J. Carroll, J. A. Pitts, K. Zhang, A. K. Pradhan, and E. E. Carpenter, J. Appl. Phys. 107, 09A302 (2010).

${ }^{22}$ M. Muruganandham, R. Amutha, B. Ahmmad, E. Repo, and M. Sillanpaa, J. Phys. Chem. C 114, 22493 (2010).

${ }^{23}$ G. Kataby, A. Ulman, R. Prozorov, and A. Gedanken, Langmuir 14, 1512 (1998).

${ }^{24}$ G. Kataby, Y. Koltypin, A. Ulman, I. Felner, and A. Gedanken, Appl. Surf. Sci. 201, 191 (1997).

${ }^{25}$ J. Dormann, A. Belayachi, J. Maknani, A. Ezzir, M. Cruz, M. Godinho, R. Cherkaoui, and M. Nogues, J. Magn. Magn. Mater. 185, 1 (1998).

${ }^{26}$ E. DeBiasi, C. A. Ramos, R. D. Zysler, and H. Romero, Phys. Rev. B 65, 144416 (2002).

${ }^{27}$ R. Zysler, H. Romero, C. Ramos, E. DeBiasi, and D. Fiorani, J. Magn. Magn. Mater. 266, 233 (2003).

${ }^{28}$ Y. Koltypin, G. Katabi, X. Cao, R. Prozorov, and A. Gedanken, J. Non-Cryst. Solids 201, 159 (1996).

${ }^{29}$ K. V. P. M. Shafi, A. Gedankenb, R. B. Goldfarb, and I. Felner, J. Appl. Phys. 81, 6901 (1997).

${ }^{30}$ K. V. P. M. Shafi, Y. Koltypin, A. Gedanken, R. Prozorov, J. Balogh, J. Lendvai, and I. Felner, J. Phys. Chem. B 101, 6409 (2010).

${ }^{31}$ K. H. J. Buschow, J. Appl. Phys. 54, 2578 (1983).

${ }^{32}$ M. Donten and Z. Stojek, J. Appl. Electrochem. 26, 665 (1996).

${ }^{33}$ M. Donten, T. Gromulski, and Z. Stojek, J. Alloys Compd. 279, 272 (1998).

${ }^{34}$ M. Naoe, H. Kazama, Y. Hoshi, and S. Yamanaka, J. Appl. Phys. 53, 7846 (1982).

${ }^{35}$ J. Dubowik, Y. V. Kudryavtsev, and R. Gontarz, Acta Phys. Pol. A 76, 331 (1989).

${ }^{36}$ M. A. M. Ibrahim, S. S. A. E. Rehim, and S. O. Moussa, J. Appl. Electrochem. 33, 627 (2003).

${ }^{37}$ K. Ounadjela, G. Suran, and F. Machizaud, Phys. Rev. B 40, 578 (1989).

${ }^{38}$ M. Naili and G. Suran, J. Magn. Magn. Mater. 135, 141 (1994).

${ }^{39}$ D. Babonneau, F. Petroff, J. L. Maurice, F. Fettar, A. Vaures, and A. Naudons, Appl. Phys. Lett. 76, 2892 (2000).
${ }^{40}$ T. Omi, H. Yamamoto, and H. L. Glass, J. Electrochem. Soc. 119, 168 (1972).

${ }^{41}$ F. Machizaud, K. Ounadjela, and G. Suran, Phys. Rev. B 40, 587 (1989).

${ }^{42}$ D. Babonneau, D. Lantiat, S. Camelio, J. Toudert, L. Simonot, F. Pailloux, M.-F. Denanot, and T. Girardeau, Eur. Phys. J. Appl. Phys. 44, 3 (2008).

${ }^{43}$ J. Briático, J.-L. Maurice, J. Carrey, D. Imhoff, F. Petroff, and A. Vaurès., Eur. Phys. J. D 9, 517 (1999).

${ }^{44}$ G. Qin, N. Xiao, B. Yang, Y. Ren, W. Pei, and X. Zhao, Acta Metall. Sin. (Engl. Lett.) 22, 415 (2009).

${ }^{45}$ B. Ravel and M. Newville, J. Synchrotron Radiat. 12, 537 (2005).

${ }^{46}$ [http://www.icsd.iqfr.csic.es/].

${ }^{47}$ B. Ravel, J. Synchrotron Radiat. 8, 314 (2001).

${ }^{48}$ B. Moraweck, A. J. Renouprez, E. K. Hlil, and R. Baudoing-Savois, J. Phys. Chem. 97, 4288 (1993).

${ }^{49}$ M. Kuhn, T. Sham, J. Chen, and K. Tan, Solid State Commun. 75, 861 (1990).

${ }^{50}$ A. Yevick and A. I. Frenkel, Phys. Rev. B 81, 115451 (2010).

${ }^{51}$ A. L. Ageev, Y. A. Babanov, V. V. Vasin, N. V. Ershov, and A. V. Serikov, Phys. Status Solidi B 117, 345 (1983).

${ }^{52}$ EXAFS spectra for the Au-capped Co NPs were recorded during the same experiment and compared to those for the uncapped Co-NPs, both with the same $t_{\mathrm{Co}}=0.7 \mathrm{~nm}$. We found that both systems have fcc structure and very close first-near-neighbor distances. Their Fourier-transformed EXAFS profiles are almost identical, even for outer coordination shells. The first coordination shell peak in the Co-Au NPs is higher in amplitude than the uncapped ones and it increases with the content of $\mathrm{Au}$ in the sample due to the high crystallinity in these noble-metal-capped particles. Then, contrary to the case of $\mathrm{W}$ deposit, noble-metal capping does not modify the crystal structure of the Co NPs.

${ }^{53}$ [http://www.sgte.org/].

${ }^{54}$ M. L. Fdez-Gubieda, A. García-Arribas, I. Orue, F. Plazaola, and J. M. Barandiarán, Europhys. Lett. 40, 43 (1997).

${ }^{55}$ A. Sadoc, D. Raoux, P. Lagarde, and A. Fontaine, J. Non-Cryst. Solids 50, 331 (1982).

${ }^{56}$ R. D. Zysler, E. DeBiasi, C. A. Ramos, D. Fiorani, and H. Romero, in Surface Effects in Magnetic Nanoparticles, edited by D. Fiorani (Springer, New York, 2005), Chap. 8, pp. 239-261.

${ }^{57} \mathrm{XMCD}$ measurements at the $\mathrm{W} L_{2,3}$ edges on these W-capped Co nanoparticle samples have been performed to determine the magnetic moment of W. It was found to be on the order of $\sim$ $10^{-3} \mu_{B} / \mathrm{W}$ at $10 \mathrm{~K}$, which is two orders of magnitude lower than the Co moment measured in the samples (see XMCD section).

${ }^{58}$ P. Hansen, in Handbook of Magnetic Materials, Vol. 6, edited by K. Buschow (Elsevier, The Netherlands, 1991), Chap. 4, pp. 289-452.

${ }^{59}$ P. Bracconi and L. Dufour, Metall. Mater. Trans. B 7, 321 (1976).

${ }^{60}$ P. Carra, B. T. Thole, M. Altarelli, and X. Wang, Phys. Rev. Lett. 70, 694 (1993).

${ }^{61}$ B. T. Thole, P. Carra, F. Sette, and G. vanderLaan, Phys. Rev. Lett. 68, 1943 (1992).

${ }^{62}$ J. Stohr, J. Electron Spectrosc. Relat. Phenom. 75, 253 (1995).

${ }^{63}$ R. Nakajima, J. Stöhr, and Y. U. Idzerda, Phys. Rev. B 59, 6421 (1999).

${ }^{64}$ K. Fauth, Appl. Phys. Lett. 85, 3271 (2004).

${ }^{65}$ A. Kleibert, K.-H. Meiwes-Broer, and J. Bansmann, Phys. Rev. B 79, 125423 (2009). 
${ }^{66}$ [http://www.nist.gov/srd/nist82.cfm].

${ }^{67}$ M. Tischer, O. Hjortstam, D. Arvanitis, J. H. Dunn, F. May, K. Baberschke, J. Trygg, J. M. Wills, B. Johansson, and O. Eriksson, Phys. Rev. Lett. 75, 1602 (1995).

${ }^{68}$ C. T. Chen, Y. U. Idzerda, H.-J. Lin, N. V. Smith, G. Meigs, E. Chaban, G. H. Ho, E. Pellegrin, and F. Sette, Phys. Rev. Lett. 75, 152 (1995).

${ }^{69}$ M. G. Samant, J. Stohr, S. S. P. Parkin, G. A. Held, B. D. Hermsmeier, F. Herman, M. van Schilfgaarde, L.-C. Duda, D. C. Mancini, N. Wassdahl, and R. Nakajima, Phys. Rev. Lett. 72, 1112 (1994).

${ }^{70}$ F. Wilhelm, P. Poulopoulos, H. Wende, A. Scherz, K. Baberschke, M. Angelakeris, N. K. Flevaris, and A. Rogalev, Phys. Rev. Lett. 87, 207202 (2001).

${ }^{71}$ R. C. O'Handley, in Modern Magnetic Materials: Principles and Applications (Wiley, New York, 2000), Chap. 11, pp. 391-431.
${ }^{72}$ A. R. Mackintosh and O. K. Andersen, in Electrons at the Fermi Surface, edited by M. Springford (Cambridge University Press, Cambridge, UK, 1980), Chap. 5, pp. 149-224.

${ }^{73}$ M. Vijayakumar and M. S. Gopinathan, J. Mol. Struct. 361, 15 (1996).

${ }^{74}$ W. A. Sucksmith and J. E. Thompson, Proc. R. Soc. London 225, 362 (1954).

${ }^{75}$ M. Spasova, U. Wiedwald, R. Ramchal, M. Farle, M. Jergel, E. Majkova, S. Luby, and R. Senderak, Phys. Status Solidi B 225, 449 (2001).

${ }^{76}$ M. Ohkoshi, N. Watanabe, and K. Tsushima, J. Magn. Magn. Mater. 113, 92 (1992).

${ }^{77}$ M. Rossignol, M. Schlenker, and Y. Samson, in Magnetism: Fundamentals, Magnetism, edited by É. Lacheisserie, D. Gignoux, and M. Schlenker (Springer, New York, 2005), Chap. 5, pp. 143 208.

${ }^{78}$ J. A. Osborn, Phys. Rev. 67, 351 (1945). 